\title{
Scaling-up improved postabortion care in Egypt: Introduction to University and Ministry of Health and Population Hospitals
}

\author{
Laila Nawar \\ Population Council \\ Dale Huntington \\ Population Council \\ Ezzeldin Osman Hassan \\ Hala Youssef \\ Nahla G. Abdel-Tawab \\ Population Council
}

Follow this and additional works at: https://knowledgecommons.popcouncil.org/departments_sbsr-rh

Part of the Family, Life Course, and Society Commons, International Public Health Commons, and the Women's Health Commons

How does access to this work benefit you? Let us know!

\section{Recommended Citation}

Nawar, Laila, Dale Huntington, Ezzeldin Osman Hassan, Hala Youssef, and Nahla G. Abdel-Tawab. 1997. "Scaling-up improved postabortion care in Egypt: Introduction to University and Ministry of Health and Population Hospitals," Asia and Near East OR/TA Project Final Report. Cairo: Population Council. 
Scaling-Up Improved Postabortion Care in Egypt: Introduction to University and Ministry of Health and Population Hospitals

\section{Final Report}


Scaling-Up Improved Postabortion Care in Egypt: Introduction to University and Ministry of Health and Population Hospitals

\title{
Final Report
}

\author{
Laila Nawar, Ph.D. \\ Dale Huntington, Sc.D. \\ Ezzeldin Osman Hassan, MD \\ Hala Yousef, MD \\ Nahla Abdel Tawab, MD, Dr.P.H.
}

\author{
Egyptian Fertility Care Society \\ Sub-contract No. 196.24A \\ The Population Council \\ Asia \& Near East Operations Research and \\ Technical Assistance Project
}

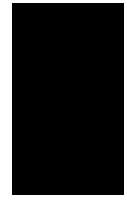

This project was supported by the Population Council's Asia \& Near East Operations Research and Technical Assistance Project. The ANE OR/TA Project is funded by the US Agency for International Development, Office of Population, under Contract Nos. DPE-C-0090-0002-10 and DPE-3030-Q-00-0023-00 Strategies for Improving Family Planning Service Delivery. 


\section{Acknowledgments}

The Egyptian Fertility Care Society conducted this study as an element of a larger program of work on improving postabortion care in Egypt that is underway with the assistance and collaboration of the Population Council's ANE OR/TA Project, and funding from USAID. UNFPA provided funds needed for obtaining supplies of Manual Vacuum Aspiration instruments and training materials.

Several agencies and individuals are associated with this program, most of whom have overlapping contributions in the different studies. The combined effect of this participation is acknowledged and we extend our apologies to any individual whose name does not appear here and has made a contribution to this study.

The strong support and assistance extended by senior staff at MOHP and NPC was critical for initiation of this research and gaining official Government of Egypt approvals to conduct the study in health care facilities.

The contributions made by the co-Principal Investigators, the staff in each of the participating hospitals and the patients, are gratefully acknowledged. Without their cooperation the quality of the results would not have been ensured.

Finally thanks are due to the staff of the Egyptian Fertility Care Society for the hard work that they put into the study during its initial stages, monitoring of the training programs, data collection, preparation and production of the final report. 


\section{Table of Contents}

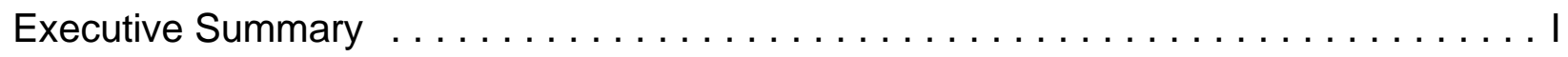

\section{Background \& Study Objectives}

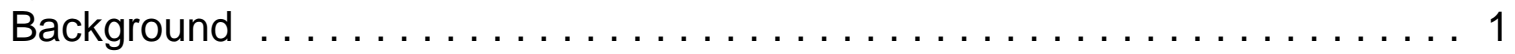

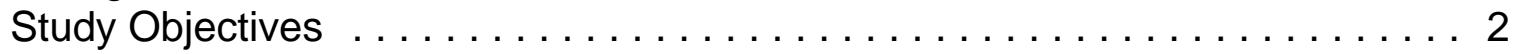

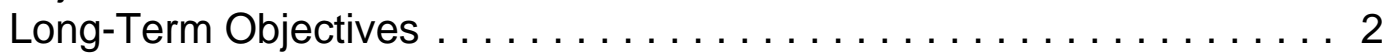

Short-Term Objectives $\ldots \ldots \ldots \ldots \ldots \ldots \ldots \ldots \ldots \ldots \ldots \ldots \ldots$

\section{Study Methodology}

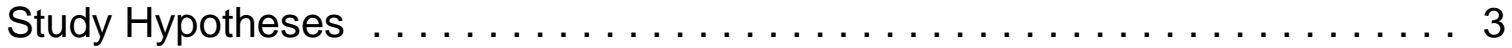

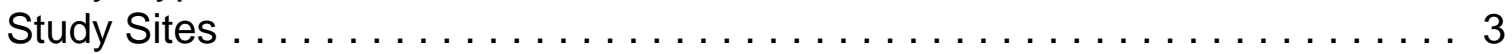

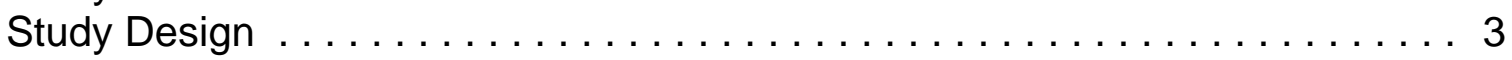

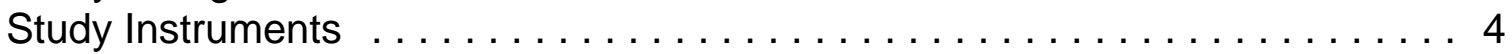

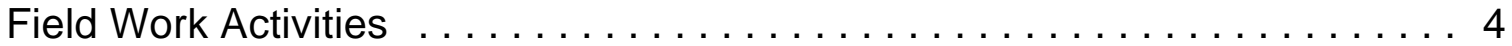

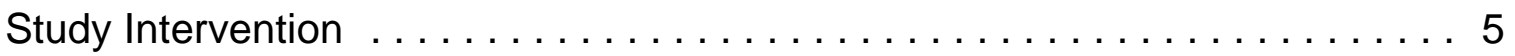

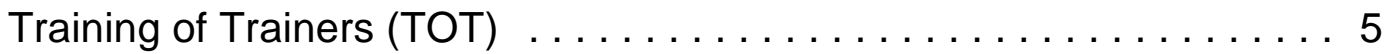

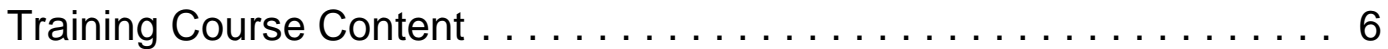

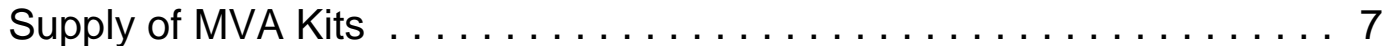

On the Job Training $\ldots \ldots \ldots \ldots \ldots \ldots \ldots \ldots \ldots \ldots \ldots \ldots \ldots$

\section{Study Findings}

Caseload \& Forms Received ..................... 9

Socio-demographic Characteristics \& Reproductive History of

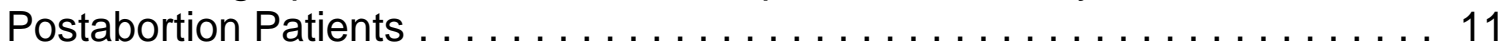

Referral and Accessibility of Health Facilities $\ldots \ldots \ldots \ldots \ldots \ldots \ldots \ldots$

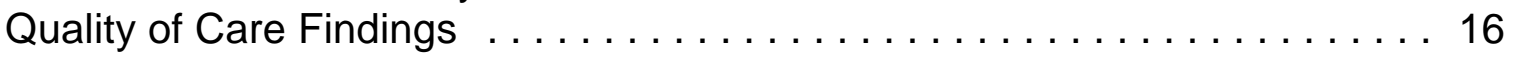

a. Physician's Technical Competence: Postabortion Care

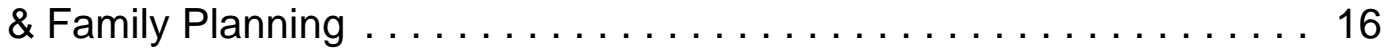

b. Interpersonal Relations ..................... 23

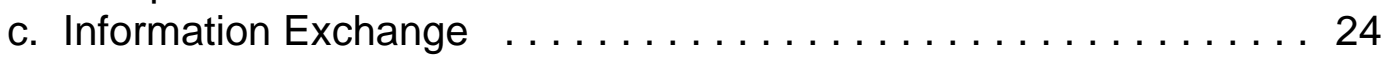

d. Utilization of Family Planning Services $\ldots \ldots \ldots \ldots \ldots \ldots 26$

\section{Overview of Findings and Conclusions}

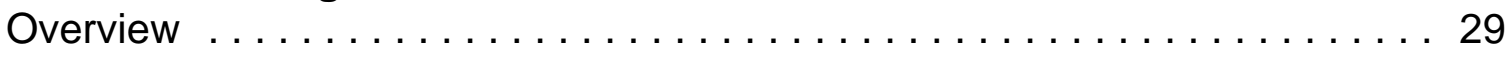

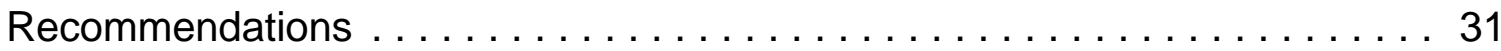

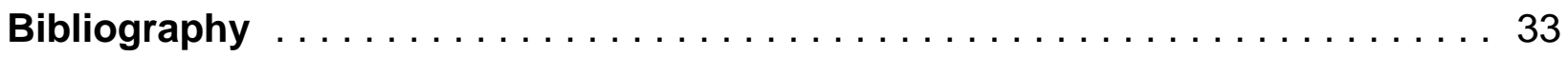




\section{List of Tables}

Table 1: Total number of forms received by type and site: pre and post test

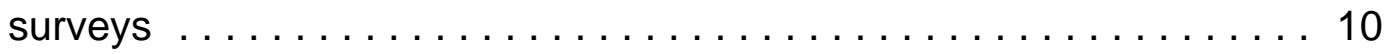

Table 2: Physicians' designation, sex and average years of experience: pre

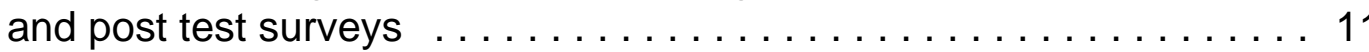

Table 3: $\quad$ Selected socio-demographic characteristics and reproductive history of postabortion patients: pre test and post test surveys . . . . 14

Table 4: $\quad$ Referral and accessibility to health facility $\ldots \ldots \ldots \ldots \ldots \ldots$

\section{List of Figures}

Figure 1: Physicians knowledge of early complications of abortion $\ldots \ldots \ldots 17$

Figure 2: Physicians knowledge about potential long-term (late) sequelae

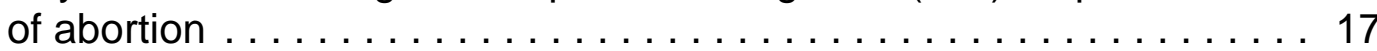

Figure 3: Physicians knowledge about warning signs of complications

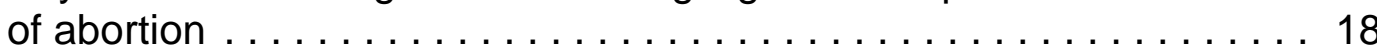

Figure 4: Type of surgical procedure used for the treatment of postabortion

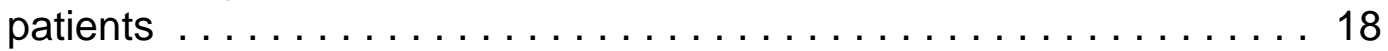

Figure 5: $\quad$ Type of anesthesia provided to postabortion patients $\ldots \ldots \ldots \ldots 19$

Figure 6: Pain experienced by postabortion patients during the surgical

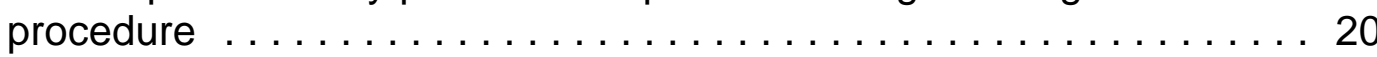

Figure 7: Physicians knowledge about timing (in weeks) when postabortion

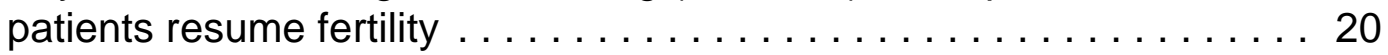

Figure 8: $\quad$ Physicians views regarding use of MVA vs. D\&C for treatment of incomplete abortion at early gestational age $\ldots \ldots \ldots \ldots \ldots 22$

Figure 9: $\quad$ MVA clinical practice: physicians' reports (post-test) $\ldots \ldots \ldots \ldots 22$

Figure 10: Physicians reports on their use of anesthesia in the past four months

Figure 11: Physicians reaction to reduce patients anxiety during the abortion procedure 
Figure 12: Selected indicators of interpersonal relations: reports of postabortion patients ...................... 24

Figure 13: Type of information physicians think that postabortion patients should know before discharge $\ldots \ldots \ldots \ldots \ldots \ldots \ldots \ldots \ldots$

Figure 14: Follow-up information provided to postabortion patients $\ldots \ldots \ldots 25$

Figure 15: Percent of postabortion patients who received information about possible complications $\ldots \ldots \ldots \ldots \ldots \ldots \ldots \ldots \ldots \ldots$

Figure 16: Postabortion family planning counseling by nurses $\ldots \ldots \ldots \ldots 26$

Figure 17: Family planning history among postabortion patients $\ldots \ldots \ldots \ldots 27$

Figure 18: Postabortion patients satisfaction with the quality of service

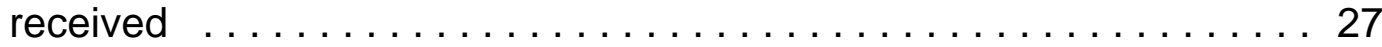




\section{STUDY TEAM}

EFCS Project Team

Professor Ezzeldin Osman Hassan

Dr. Farag Rizk Hassan

Mrs. Naglaa El Nahal

Dr. Hala Youssef

Mr. Ahmed Abdallah

\section{Population Council Team}

ANE OR/TA Project Technical Support

Dr. Dale Huntington

Dr. Laila Nawar

Dr. Nahla Abdel Tawab

Ms. Sahar Hegazi

\section{Consultants}

Dr. Nahid Toubia

Dr. Abdel Aziz El Shoubary

Dr. Mohammed Naguib

Dr. Maaly Guimie

\section{Field Co-Investigators}

Heads of OB/GYN Department

Professor Ibrahim Azab

Professor Samy Saed

Professor Mohamed El Shafei

Professor Sayed Kafafi

\section{Field Coordinators}

\section{OB/GYN Specialists}

Dr. Sameh Saadeldin

Dr. Hesham Shalan

Dr. Mohamed El Hini

Dr. Hassan Abu Elnoor

Dr. Osama El Attar

Dr. Mahdy El Feki

Dr. Farghal Salaheldin

Dr. Adel El Meniawy

Dr. Mohamed Naguib

Dr. Mohamed El-Khatib

\section{Trainers}

\section{OB/GYN Specialists}

Professor Yosri Khamis
Principal Investigator

Advisor

Program Officer

Project Coordinator

Data Analyst

Deputy Director

Host Country Advisor

Country Fellow

Communications Officer

Rainbow

El Galaa Maternity Hospital

Cairo University

AVSC, Int.

Shatby University Hospital Shatby University Hospital Mansoura University Hospital Menia University Hospital

Shatby University Hospital Mansoura University Hospital

Menia University Hospital

Damanhour Teaching Hospital

Mansoura General Hospital

Menia General Hospital

Abu Korkas Hospital

Samalout Hospital

Bani Mazar Hospital

Maghagha Hospital

Shatby University Hospital 


\author{
Dr. Sameh Saadeldin \\ Dr. Hassan Mansour \\ Dr. Osama El Ashkar \\ Dr. Tarek Karkour \\ Dr. Wafaa Abu El Einin \\ Professor Mohamed El Shafie \\ Dr. Lotfy Sherif \\ Dr. Abdel Meguid Mashaly \\ Dr. Hesham Shaalan \\ Dr. Mohamed El Negiry \\ Professor Sayed Kafafi \\ Dr. Mohamed El Hini \\ Dr. Essam Ibrahim \\ Dr. Mohamed Hani \\ Dr. Amgad Osman \\ Dr. Hassan Abu Elnoor \\ Dr. Samya Assad \\ Dr. Hosni El Feshawy \\ Dr. Taha Sharkawy \\ Dr. Osama El-Attar \\ Dr. Abdel Halim Ragab \\ Dr. Kamal Hussein \\ Dr. Ezzat Moustafa \\ Dr. Saed El Sharkawy \\ Dr. Mahdy El Feki \\ Dr. Abdallah Abdel Ghani \\ Dr. Abu Bakr Ahmed \\ Dr. Shantoury Bayoumi \\ Dr. Sanaa El Mankabady \\ Dr. Farghal Salaheldin \\ Dr. Aly Moharam \\ Dr. Maha Korolos \\ Dr. Attalah Shehata \\ Dr. Ahmed Montasser \\ Dr. Adel El Meniawy \\ Dr. Osama Abbas \\ Dr. Hegazi Mohamed Abdel Maksoud \\ Dr. Ibrahim Zaki Saleh \\ Dr. Abdu Hassan Abdu \\ Dr. Mohamed Naguib \\ Dr. Hamada Ragab \\ Dr. Islam Ahmed \\ Dr. Ezzat Helal \\ Dr. El Zaeem Ismail \\ Dr. Mohamed El Khatib
}

Shatby University Hospital Shatby University Hospital Shatby University Hospital Shatby University Hospital Shatby University Hospital Mansoura University Hospital Mansoura University Hospital Mansoura University Hospital Mansoura University Hospital Mansoura University Hospital Menia University Hospital Menia University Hospital Menia University Hospital Menia University Hospital Menia University Hospital Damanhour Teaching Hospital Damanhour Teaching Hospital Damanhour Teaching Hospital Damanhour Teaching Hospital Mansoura General Hospital Mansoura General Hospital Mansoura General Hospital Mansoura General Hospital Mansoura General Hospital Menia General Hospital Menia General Hospital Menia General Hospital Menia General Hospital Menia General Hospital Abou Korkas Hospital Abou Korkas Hospital Abou Korkas Hospital Abou Korkas Hospital Abou Korkas Hospital Samalout Hospital Samalout Hospital Samalout Hospital Samalout Hospital Samalout Hospital Bani Mazar Hospital Bani Mazar Hospital Bani Mazar Hospital Bani Mazar Hospital Bani Mazar Hospital Maghagha Hospital 
Dr. Attia Abdel Hakim

Dr. Sobhi Habib

Dr. Mourad Adly Guindy

Dr. Amir Habib
Maghagha Hospital

Maghagha Hospital

Maghagha Hospital

Maghagha Hospital 


\section{EXECUTIVE SUMMARY}

This research launched an expansion of improved postabortion care services introduced through a small pilot study in 1994. This study aims at institutionalizing improved postabortion medical care and counseling procedures as well as increasing use of family planning by postabortion patients. The case management protocol was based on the use of manual vacuum aspiration (MVA) under local anaesthesia (instead of the customary practice of Dilatation and Curettage (D\&C) technique performed under general anaesthesia). Training of physicians also included a counseling component to reduce patients anxiety and concerns regarding their future health and referral for family planning and other related health services. The study's intervention also improved nurses' skills in infection control procedures and counseling skills with postabortion patients. An additional element to this study investigated costs associated with the introduction of improved postabortion services. However, findings on this cost element will be presented in a forthcoming separate report.

A pre-test / post-test, no control group study design was utilized to measure the effect of the study intervention on providers' knowledge and practices with regard to the management of postabortion cases and selected patient outcome measures. The study was conducted in the OB/GYN wards of ten hospitals in the four governorates of Menia, Dakahlia, Alexandria and Behira including three universities and seven Ministry of Health and Population (MOHP) facilities. The study's pre-and-post intervention surveys utilized direct interviews with 501 physicians and 574 nurses working in the OB/GYN wards, in addition to interviews with 1,005 consenting postabortion patients prior to their discharge and abstracts of 1,036 medical records.

A five day training program was conducted at each of the three University hospitals to launch the study's intervention. Five senior physicians from each of the study sites were trained as master trainers in the new clinical practices and counseling techniques for improved postabortion care. This training was followed by four months of on-the-job training in which the master trainers trained other physicians and nurses in their respective hospitals. The training course included both theoretical and practical 
components and was conducted in a participatory manner. A special training course was also provided to nurses during the same period that emphasized their role in counseling and infection control procedures.

The training was based on IPAS materials and was supplemented by a one day training session on counseling modules in reproductive health care and contraceptive use. A modified module on patient-provider interaction was presented to make it applicable to the audiences. An original protocol for the management of incomplete abortion was also developed. It is important to note that the MVA kits and training materials were procured from IPAS with funds from the UNFPA Egypt country office. The UNFPA grant provided enough MVA kits for the study period and an additional 18 months, when it is anticipated that the MVA supply will be ensured by the Egyptian Ministry of Health and Population.

Overall, the pre-and-post test samples of patients did not show any significant differences in terms of their socio-demographic characteristics. The mean gestational age of the index pregnancy (that was the cause of the postabortion hospitalization) was slightly higher for the pre-test patients (11.4 weeks) than the post-test (10.5 weeks). This was due to the pre-test period including a few late gestational age cases. Close to one half of the postabortion patients reported a desire for more children and the same proportion reported having ever used a contraceptive method in both pre-and-post-test surveys.

WHO guidelines for classifying hospitalized postabortion patients were applied to this study. Approximately one half of all the postabortion cases in both the pre-test and the post-test surveys can be classified as spontaneous abortions (55\%), one third of the cases were classified as possibly induced and the remainder were classified as either probably or certainly induced. This classification is a rough index of the causes of abortion and should be interpreted cautiously.

The intervention had a substantial effect on changing the surgical procedure for ensuring the complete evacuation of the uterine contents. Prior to the study intervention, about $98 \%$ of postabortion patients were managed with the D\&C 
technique. This percentage dropped to $40 \%$ in the post-test while the percentage of patients treated by MVA increased to $57 \%$. Results showed a drop in the use of general anesthesia from $85 \%$ in the pre-test to $51 \%$ in the post-test. These changes in the clinical practices were met by satisfaction of physicians and acceptance of the MVA technique. Three quarters of physicians perceived lower complications with the use of MVA versus the use of D\&C. About $61 \%$ stated that MVA is easier to use while $56 \%$ mentioned that it is more effective.

The study also examined physicians' technical knowledge related to postabortion care. Findings reflected that prior to the study physicians had deficient and incomplete knowledge about early complications, long term sequelae of abortion as well as warning signs of complications after having an abortion or miscarriage. After the study intervention modest improvements in physicians' factual knowledge were observed. Knowledge about the resumption of fertility substantially increased as the percent of physicians reporting the correct time increased from $4 \%$ in the pre-test to $43 \%$ in the post-test.

\section{Results indicated a positive effect on the interpersonal communication} processes between the medical staff and postaboation patients. Patients who reported that they were received by a friendly provider increased from $56 \%$ in the pretest to $82 \%$ in the post-test. Also, fewer patients in the post-test reported that they were blamed by service providers for their medical condition than in the pre-test. However, information provided by physicians to postabortion patients on the possible complications of abortion, aspects of their health care and important follow up information had only a modest increase when the results of the pre-and-post-test were compared. It is worth noting that the waiting time between admission and receiving medical care decreased significantly in the post-test.

The study had a positive effect on postabortion patients' intention to begin using a contraceptive method. About one third of the patients had the intention to begin using a method in the pre-test which increased to about one half in the post-test. Among those patients who decided to use a contraceptive, $22 \%$ in the pre-test and 
$15 \%$ in the post-test were provided with a method before discharge. In addition, the percentage of patients having the intention to use a family planning method within two weeks of discharge increased from $24 \%$ in the pre-test to $36 \%$ in the post-test.

Based on the findings of the present study it is recommended that steps be taken on both the policy and program levels to institutionalize the use of MVA under local anesthesia and improved counseling procedures in both the public and the private sectors. Effective mechanisms need to be developed for integrating postabortion services with family planning as well as other reproductive health services. Sustainable supplies of MVA kits should be made available at affordable prices. This highlights the role of the Ministry of Health and Population in securing the necessary drugs and chemicals needed for pain and infection control procedures for use with MVA instruments. Finally a protocol for the use of MVA as part of the accepted standards needs to be incorporated in medical curricula and a high emphasis needs to be placed on training nurses and other para-medical staff involved in postabortion care. 


\section{Background}

Abortion constitutes an important health problem for women in many countries. Although accurate data on the impact of unsafe abortion on maternal health is lacking, recent $\mathrm{WHO}$ estimates indicate that up to $15 \%$ of pregnancy related mortality is due to abortion (WHO, 1995).

In Egypt, the legal, religious and societal restrictions on abortion have constrained the provision of high quality emergency health care services provided to postabortion patients, including linkages with family planning services. In most cases, medical training and clinical practices are inadequate. The standard treatment of incomplete abortion is dilatation and curettage (D\&C) which is usually performed in the operating theater under general anesthesia. This places the patient at risk for a number of complications, including hemorrhage and uterine perforation. It also poses a significant drain on scarce health care resources. Moreover, the management of psychological stress associated with abortion and providing adequate information and counseling of postabortion patients as a humane and preventive measure to avoid subsequent unplanned pregnancies is nearly non-existent in most hospitals.

In 1994 a small scale pilot study was conducted by the Egyptian Fertility Care Society (EFCS) and the Population Council's ANE OR/TA Project (1995) to improve postabortion care in Egypt. The study was conducted in Menia University and EI Galaa Hospitals. The study intervention included an intensive training program for physicians on the use of Manual Vacuum Aspiration instruments (MVA) under local anesthesia. Physicians were also instructed in infection control practices and counseling postabortion patients about their medical condition and family planning. The study findings reflected significant improvements in the care of postabortion patients.

The highly positive impact of this pilot study led the Population Council's ANE OR/TA Project to develop a comprehensive Introduction Program for Improved Postabortion Care in Egypt which was approved by the Government of Egypt. One major component of this program is to expand the improved postabortion care services based on the experience gained in the pilot study. Activities of this component were 
implemented in collaboration with the Egyptian Fertility Care Society.

An additional component of the expansion program also includes a cost study that investigates the quantity and type of resources used and costs associated with treatment of postabortion patients (both before and after use of MVA instruments). Reducing the cost associated with treating incomplete abortion would be clearly advantageous in a setting of scarce resources and would contribute to subsequent expansion activities. Results from this cost study will be released in a forthcoming separate report.

\section{Study Objectives}

\section{Long Term Objective}

The study will contribute to decreased risk of maternal mortality and morbidity associated with abortion through expanding improved postabortion clinical care and addressing contraceptive needs of women who are at risk of an unwanted pregnancy.

\section{Short Term Objectives}

1. To institutionalize improved postabortion medical services and counseling procedures in selected Egyptian University Hospitals, Teaching and other Public Sector hospitals including increased use of family planning services by postabortion patients, based on the experience gained in the pilot study.

2. To estimate selected costs associated with the introduction of improved postabortion services.

3. To provide useful guidelines for enhanced program management and informed policy development with respect to training modules and clinical protocols and practices. 


\section{Study Hypotheses}

The following hypotheses were tested by this study:

1. Use of Manual Vacuum Aspiration for treating postabortion patients instead of use of $D \& C$ will have a positive impact on the quality of the health care provided to women.

2. Providing counseling to postabortion patients after the procedure and before discharge will minimize patients anxiety and concerns about their current and future health situation and will promote their knowledge, attitudes and intention to adopt family planning.

3. Use of Manual Vacuum Aspiration instruments will be positively associated with a lower use of hospital resources and lower costs than the current use of D\&C for treatment of incomplete abortion.

\section{Study Sites}

The following sites have been selected for the present study: the three University hospitals in Menia, Alexandria and Mansoura, and seven General, Teaching and District hospitals with linkages to the above three university hospitals:

- Menia General Hospital, Abou-Korkas District Hospital, Samalut District Hospital, Bani-Mazar District Hospital, and Maghagha District Hospital (with links to the University of Menia)

- Damanhour Teaching Hospital, (with links to the University of Alexandria)

- Mansoura General Hospital, (with links to the University of Mansoura).

The three University hospitals selected have good physical infrastructure, relatively high incomplete abortion caseload and good experience in institutionalizing medical research skills. Their staff also have high potential for providing training, follow-up and technical assistance to staff members of other sites included in the study.

\section{Study Design}

The study utilized a pre-test, post-test, no comparison group study design to examine the intervention's effect on improving postabortion care as stated in the study hypotheses. Immediately after completion of data collection in the pre-test phase, an 
intervention was implemented (described below ). A four month study period elapsed between the pre and post test phases during which the desired changes in clinical practice were followed rigorously by a core of staff trainers assigned at the ten study sites.

\section{Study Instruments}

The first two hypotheses were assessed using the following three data collection methods in both the pre-test and post-test surveys:

a) Interviews with hospital staff: These interviews were conducted with all medical providers who are involved in providing care to postabortion patients, including physicians, nurses and social workers.

b) Interviews with Patients: Exit interviews were conducted with all consenting postabortion patients during a continuous thirty day period to collect information on the quality of care they received. Informed consent of women was secured before undertaking the interviews.

c) Abstracts of Medical Records: These forms describe all patient's medical status upon admission, surgical procedure and medications used as well as selected socio-demographic and reproductive history profile, during a continuous thirty day period.

The three study instruments are based on those used in the pilot study (1995). Instruments were revised and updated to reflect changes in the training curriculum as well as lessons learned in the analysis of the pilot study.

\section{Field Work Activities}

\section{Qualifications of Data Collectors}

Individuals selected as data collectors included both physicians and social workers. Physicians not associated with the study and not from the hospital where the interviews were conducted were trained to conduct interviews with clinic staff and to prepare abstracts of medical records. In order to perform these tasks accurately, the physicians were selected from OB/GYN specialists (or residents) with good experience in the management of postabortion cases. Female social workers and nurses with good experience in social science research conducted the exit interviews with the 
postabortion patients and with nurses and social workers in each hospital.

\section{Training and Pre-Testing of Data Collection Instruments}

A training program for data collection was organized at Menia and Alexandria University hospitals in early September, 1996 prior to the pre-test survey .A refresher training session was held at each of the university hospitals early in March, 1997 prior to the post-test survey .

The original training was a five-day course and highlighted the importance and objectives of the study and focused on both theoretical and practical aspects. Training of data collectors was done separately for social workers and physicians and involved intensive role playing. Study instruments were pre-tested in hospitals other than those included in the study (Bani Sewef General Hospital for the training program organized in Menia and Dar Ismail General Hospital in Alexandria for the training program organized in Alexandria).

\section{Data Collection}

The pre-test data collection period was a continuous 4 week period (beginning on

September 9, 1996), and the post-test data collection period was also 4 weeks (beginning on March 14, 1997). Completed data collection

The study was based on a pre-test, post-test no comparison group design. Three data collection instruments were utilized:

$\checkmark$ Interviews with hospital staff $\checkmark$ Interviews with patients $\checkmark$ Abstracts of medical records instruments were sent to EFCS office in Cairo on weekly basis. Regular monitoring of all data collection activities was rigorously enforced.

\section{Data Entry and Analysis}

Data entry and analysis for both pre and post test surveys were done centrally at the EFCS office by a well trained team using SPSS PC software package.

\section{Study Intervention}

\section{Training of Trainers (TOT)}

Changes in the clinical management of postabortion patients were introduced through an intensive 5-day training program in each university hospital attended by 5 
senior OB/GYN specialists from each participating hospital. The training strategy focused on developing a cadre of master trainers who could then train their colleagues and junior physicians and provide daily on-site supervision and follow-up. The training course was conducted in October 1996 in a participatory manner with both theoretical and practical components. It included demonstrations of the technique of manual vacuum aspiration, description of components/mode of action of the MVA instruments, infection control procedures and supervised practice in the surgical theaters of the three university hospitals.

An experienced international medical consultant, Dr. Nahid Toubia assisted EFCS in the development and conduct of the training course. The three Co-Principal Investigators, who are the heads of OB/GYN departments in the three medical schools included in the study, as well as other senior faculty staff participated in the sessions as lecturers and discussion leaders.

\section{Training Course Content}

The course was based on IPAS training materials for the introduction of MVA instruments, (Yorde, et.al, 1993), which were reviewed by the principal investigator of the study as well as the co-principal investigators for adaptation of use within the Egyptian context. The course was supplemented by a one day training session on counseling modules in reproductive health care and contraceptive use conducted by Dr. Maaly Guimie, a highly experienced trainer who is well versed in this area. A module on patient-provider interaction was presented in a modified version to suit the environment of training and to make it applicable to the audience. The trainees responded very well to this part of training which deals more with behavioral and psychological issues despite their lack of exposure to many of the concepts introduced. In fact there was a clear eagerness to understand more and to discuss frankly the obstacles which prevent them from giving more humane and individualized care to their clients. 
An original protocol for the management of incomplete abortion was developed for use in the ten sites participating in the study based on IPAS materials. It includes the treatment of mild, moderate and severe abortion complications ( both pre and postoperatively ), controlling the pain of an anxious and ill patient, and family planning counseling. The use of vacuum aspiration combined with an emphasis on a holistic approach to patient care was a new concept for many of the physicians who were more familiar with higher level interventions and more specialized treatments.

The psychological aspects of learning a new clinical procedure were addressed as a move towards higher service quality and excellence. In this regard, use of the MVA procedure was presented as a technique that requires the sensitivity of a plastic or vascular surgeon. There was also a clear consensus on the need to provide family planning counseling and to assist patients achieving their reproductive intentions.

A special training course was conducted for nurses involved in providing care to postabortion patients. Emphasis was placed on their role in counseling and comforting postabortion patients, instruments disinfection and storage, and preparing MVA kits needed for daily procedures.

In general, the receptivity of training was high. However, some participants showed some resistance related to infection control requirement and pain control procedures. Some of the resistance was due to personal habits and practices that have been used for several years. It was also due to lack of availability of drugs or chemicals needed (e.g. Cidex), especially at some district hospitals.

\section{Supply of MVA Kits}

MVA kits (double valve syringes, flexible Karman cannulae sized 6-12 mm, and syringe replacement parts) were procured from IPAS with funds from a UNFPA, Egypt grant to the Population Council. The ten study hospitals were provided with enough 
supplies of syringes and cannulae of different sizes for training purposes as well as for use over a period of at least 18 months. This supply of MVA kits is meant to serve as a bridging stock until a system for continuous supply of MVAs is institutionalized by the Egyptian Ministry of Health and Population.

Procurement and distribution of MVA instruments have been carefully monitored by the Population Council office in Cairo. The Population Council maintained stock inventory and disbursement forms for each hospital. These forms were used to carefully and concisely record the movement of all MVA instruments from initial orders, receipt in Cairo, until reaching each participating hospitals. Moreover, the site coordinator in each of the study hospitals submits a monthly inventory report that shows the number of instruments that were available at the beginning of the month, the number used and the balance remaining at the end of the month.

\section{On the Job Training}

For a period of four months, master trainers in the ten study hospitals supervised a continuous process of on the job training for other colleagues and junior physicians. Ensuring the provision of improved postabortion care based on the established protocols for management of incomplete abortion cases was the main objective. During this four month period frequent site visits (at least once weekly) were made to each of the ten hospitals by the study coordinator, a skilled OB/GYN consultant as well as EFCS and Population Council staff. These site visits were to follow up on the implementation and enforcement of the new clinical procedures and counseling practices. A site visit check list was developed to monitor the implementation of the components of the intervention. In addition, master trainers from university hospitals were assigned the responsibility of visiting MOHP hospitals located in their area on a weekly basis to spot check on the implementation of the intervention and to answer staff questions. In addition they were required to submit follow-up reports to EFCS on the progress of training at each site. 


\section{Case Load and Forms Received}

Table (1) shows the sampling results for each type of data collection instruments during the pre- and post-test phases of the study by site. Data was collected from a total of 1,036 medical records, 1005 patient exit interviews, 501 physician interviews, and 574 nurse interviews both in the pre- and post-test surveys.

The table indicates that Shatby University Hospital received the highest case load of postabortion patients both in the pre and post-test surveys. By contrast, Mansoura General and Abou-Korkas District hospitals reported the lowest case loads. It is known that the former hospital receives postabortion patients and delivery cases only 2 days a week (Mansoura University Hospital receives these patients on the other days of the week) and Abou-Korkas generally receives a low postabortion case load.

Discrepancies between the number of completed pre and post-test questionnaires of Patient Exit Interview can be attributed to differences in caseload during the two data collection periods (one month each). In addition, a few postabortion patients for whom medical records were completed refused to go through Patient Exit Interview (2.7\% refusal rate for post-test interviews). This explains the discrepancies between the number of Medical Records and that of Patient Exit Interviews. As for differences in numbers of medical staff interviewed in pre and post test phases, these are attributed to both changes in the number of medical staff in charge at time of data collection. 
Table 1

Total Number of Forms Received by Type and Site : Pre and Post-test Surveys

\begin{tabular}{|c|c|c|c|c|c|c|c|c|}
\hline \multirow{3}{*}{ Site } & \multicolumn{8}{|c|}{ 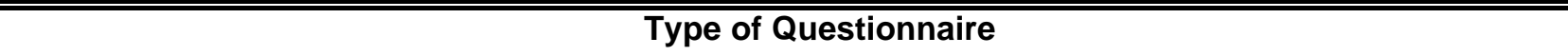 } \\
\hline & \multicolumn{2}{|c|}{ Medical Record } & \multicolumn{2}{|c|}{ Physician Interview } & \multicolumn{2}{|c|}{ Nurse Interview } & \multicolumn{2}{|c|}{ Patient Exit Interview } \\
\hline & Pre-test & Post-test & Pre-tes & Post-test & Pre-te & ost-test & & Post-test \\
\hline Menia University Hospital & 36 & 30 & 25 & 22 & 18 & 13 & 35 & 29 \\
\hline Mansoura University Hospital & 53 & 56 & 28 & 32 & 39 & 37 & 53 & 56 \\
\hline Shatby University Hospital & 195 & 142 & 50 & 50 & 126 & 106 & 199 & 142 \\
\hline Damanhour Teaching Hospital & 60 & 73 & 22 & 24 & 31 & 28 & 50 & 73 \\
\hline Menia General Hospital & 39 & 56 & 35 & 15 & 30 & 16 & 38 & 53 \\
\hline Mansoura General Hospital & 14 & 24 & 39 & 47 & 18 & 24 & 14 & 24 \\
\hline Maghagha District Hospital & 35 & 41 & 14 & 16 & 25 & 19 & 37 & 41 \\
\hline Abu-Korkas District Hospital & 16 & 31 & 17 & 12 & 7 & 6 & 14 & 21 \\
\hline Bani Mazar District Hospital & 39 & 33 & 15 & 16 & 7 & 7 & 36 & 31 \\
\hline Samalout District Hospital & 32 & 31 & 10 & 12 & 10 & 7 & 32 & 27 \\
\hline Total & 519 & 517 & 255 & 246 & 311 & 263 & 508 & 497 \\
\hline
\end{tabular}


Table 2

Physicians' Designation, Sex and Average Years of Experience:

Pre and Post-test Surveys

\begin{tabular}{||l|c|c||}
\hline \multicolumn{1}{|c|}{ Variable } & $\begin{array}{c}\text { Percentage } \\
\text { Pre-test (n=255) }\end{array}$ & $\begin{array}{c}\text { Percentage } \\
\text { Post-test (n=247) }\end{array}$ \\
\hline \hline Designation of Physician & 40.0 & 54.1 \\
OB/GYN Specialist & 43.9 & 41.1 \\
Resident & 16.1 & 4.8 \\
Other & 62.6 & 70.1 \\
\hline Sex & 37.4 & 29.9 \\
Male & 10.7 & 10.6 \\
Female & 6.2 & 6.6 \\
\hline Mean Years of Experience & & \\
Years of practicing medicine & \multicolumn{2}{|l}{} \\
Years of service at hospital & \multicolumn{2}{|l}{} \\
\hline \multicolumn{2}{|l}{ Souce:Physian' }
\end{tabular}

Source : Physicians' Interviews

Differences between pre and post-test were not significant for all variables shown.

Table (2) shows the total number of physicians who were interviewed during the pre and post-test phases of the study by their qualifications, sex, and years of experience. Because of transfers and staff rotation some physicians interviewed in the pre test were not included in the second data collection phase. About two fifths of the physicians interviewed in the pre-test survey (and about one half in the post test) were $\mathrm{OB} / \mathrm{GYN}$ specialists. One third of physicians in both phases of the study were females. The average length of service at the study hospitals was approximately six years in the pre-test and six and a half years in the post-test (the difference was not statistically significant). The average number of years of practicing medicine was approximately 11 years during both survey intervals indicating that the interviewed physicians have more or less the same experience.

\section{Socio-Demographic Characteristics and Reproductive History of Postabortion Patients}

Table (3) presents selected socio-demographic and reproductive characteristics 
of postabortion patients. Data shown in this table are derived from two data sets, the Patient Exit Interviews and the Medical Records. The criteria for identifying the data set used relied on whether the information needed for analysis required the physician's clinical examination or the patient's self reporting.

Data of this table show that the majority of postabortion patients fall within the broad age group of 20-39 years. Mean age was slightly higher in the pre-test survey (28.5 years) than in the post-test survey (27.9 years), however the difference was not statistically significant. Though illiteracy was higher in the post-test group (60\%) compared to that in the pre-test (55\%) the difference was not statistically significant. It should be noted that patients with higher education (high school/university or more) are well represented, (about $25 \%$ in the pre-test survey and $21 \%$ in the post-test group) and that most of the patients are housewives.

Though there was an increase in the percentage of housewives in the post-test survey group ( $88 \%$ compared to about $84 \%$ in the pre-test) and a decrease in the percentage of working women (16.1 in pretest phase to 11.7 in post-test phase), the differences were not statistically significant.

In both the pre and post-test surveys it is apparent that the majority of postabortion patients have a parity of 3 or less. It is interesting to note that about $21 \%$ in the pre-test and $25 \%$ in the post-test are nulliparous women.

Data on desire for more children and family planning experience seem to indicate that in both pre and post test surveys, a large proportion of postabortion patients have not yet achieved their fertility desires and are less likely to have used a contraceptive method when these results are compared to the 1995 Egyptian Demographic Health Survey (EDHS) data. Comparable data from EDHS, 1995 indicate that $34.6 \%$ of married women want more children and $70.4 \%$ ever used a contraceptive method. About $53 \%$ of the postabortion patients in the pre-test and $57 \%$ in the posttest want more children. Fifty-three percent of patients in pre-test reported ever use of a contraceptive method compared to $48.1 \%$ in the post-test. There was no statistical significance denoting a difference between women interviewed in pre or post-test 
phases in this regard.

Based on women's self-reports, table (3) shows that $43 \%$ of the index pregnancies in pre-test and $42 \%$ in the post-test were unplanned. Among those unplanned pregnancies, $46 \%$ in the pre-test and about $41 \%$ in post-test survey were unwanted. There was a statistically significant difference in the average gestational age of the pregnancy just lost between the pre-test (11.4 weeks) and the post test (10.5 weeks). The proportion of patients with an aborted pregnancy of gestational age less than or equal 12 weeks increased from $81.3 \%$ during the pre-test to $87.0 \%$ during the post-test and the difference was also statistically significant.

Finally, table (3) categorizes postabortion patients using the WHO Task Force Classification Protocol for classifying morbidity related to abortion using hospital based survey results (WHO, 1987). This protocol provides guidelines for classifying abortion cases according to criteria provided by the patient herself (wantedness of the pregnancy, use of contraception), and/or complications related to induced abortion as recorded upon admission (e.g. foreign body in the genital tract or trauma or uterine sepsis).

As shown in the table, approximately one half of the post-abortion cases in both the pre and the post-test surveys can be classified as spontaneous abortions (55\%). One third of the cases (35\% in pre and $33 \%$ in post-test) are A large proportion of postabortion patients have not yet achieved their fertility desires and are less likely to have used a contraceptive method when these results are compared to the 1995 Egyptian Demographic and Health Survey (EDHS) classified as possibly induced and the remainder are classified as either probably or certainly induced. It may be noted that more postabortion patients were classified as "certainly induced" in the post-test than in the pre-test surveys ( $12 \%$ vs. $8.1 \%$, respectively). 
Table 3

Selected Socio-Demographic Characteristics and Reproductive History of Postabortion Patients:

Pre-test and Post-test Surveys

\begin{tabular}{|c|c|c|}
\hline Characteristic & $\begin{array}{c}\text { Percentage } \\
\text { Pre-test }\end{array}$ & $\begin{array}{c}\text { Percentage } \\
\text { Post-test }\end{array}$ \\
\hline $\begin{array}{l}\text { Age } \\
<20 \\
20-29 \\
30-39 \\
40+ \\
\text { Mean Age }\end{array}$ & $\begin{array}{c}8.5 \\
45.5 \\
39.3 \\
6.7 \\
28.5\end{array}$ & $\begin{array}{c}9.9 \\
50.1 \\
33.6 \\
6.4 \\
27.9\end{array}$ \\
\hline $\begin{array}{l}\text { Education } \\
\text { Illiterate } \\
\text { Primary/Preparatory } \\
\text { Secondary and over }\end{array}$ & $\begin{array}{l}55.0 \\
20.3 \\
24.7\end{array}$ & $\begin{array}{l}60.0 \\
18.9 \\
21.1\end{array}$ \\
\hline $\begin{array}{l}\text { Employment Status } \\
\text { House Wife } \\
\text { Working } \\
\text { Student }\end{array}$ & $\begin{array}{c}83.7 \\
16.1 \\
0.2\end{array}$ & $\begin{array}{c}88.1 \\
11.7 \\
0.2\end{array}$ \\
\hline $\begin{array}{l}\text { Parity } \\
0 \\
1-3 \\
4+ \\
\text { Mean Parity } \\
\text { Mean Previous Still Births } \\
\text { Mean Previous Miscarriages }\end{array}$ & $\begin{array}{l}21.3 \\
50.3 \\
28.4 \\
2.5 \\
0.15 \\
0.70\end{array}$ & $\begin{array}{c}24.7 \\
48.5 \\
26.8 \\
2.4 \\
0.04 \\
0.68\end{array}$ \\
\hline $\begin{array}{l}\text { Want More Children } \\
\text { No } \\
\text { Yes } \\
\text { Depends on God /Husband } \\
\text { Other answers }\end{array}$ & $\begin{array}{c}43.1 \\
53.1 \\
3.8 \\
0.0\end{array}$ & $\begin{array}{c}38.4 \\
57.3 \\
3.6 \\
0.7\end{array}$ \\
\hline $\begin{array}{l}\text { Family Planning Experience } \\
\text { Percent ever used contraceptives } \\
\text { Percent used contraceptives at } \\
\text { Time of Index Pregnancy }\end{array}$ & $\begin{array}{l}52.8 \\
18.9\end{array}$ & $\begin{array}{l}48.1 \\
16.5\end{array}$ \\
\hline $\begin{array}{l}\text { Type of Abortion }{ }^{\text {a }} \\
\text { Certain } \\
\text { Probable } \\
\text { Possible } \\
\text { Spontaneous }\end{array}$ & $\begin{array}{l}8.1 \\
1.5 \\
35.6 \\
54.7\end{array}$ & $\begin{array}{c}12.0 \\
1.0 \\
32.5 \\
54.5\end{array}$ \\
\hline $\begin{array}{l}\text { Mean Gestational Age }{ }^{\text {a }}(\text { Weeks })^{\star} \\
\text { Mean Uterine Size }{ }^{\text {a }}(\text { Weeks })^{\star} \\
\text { Percent with aborted pregnancy }{ }^{\star a} \\
\text { of gestational age }<12 \text { weeks }\end{array}$ & $\begin{array}{l}11.37 \\
10.17 \\
81.3 \\
\end{array}$ & $\begin{array}{c}10.5 \\
9.6 \\
87.0\end{array}$ \\
\hline $\begin{array}{l}\text { Index Pregnancy }^{\mathbf{a}} \\
\text { Unplanned } \\
\text { Unwanted }^{\mathrm{b}}\end{array}$ & 519) ${ }_{(\mathrm{n}=223)}^{43.0} 45.7^{(\mathrm{n}=}$ & $\begin{array}{ll}41.7 & (n=515) \\
40.6 & (n=212)\end{array}$ \\
\hline
\end{tabular}

Source : - Patients' Exit Interviews 


\author{
${ }^{\text {a }}$ Medical Record \\ ${ }^{b}$ For pregnancies that were unplanned \\ - * $p<0.001$ \\ - Unless otherwise specified differences between pre and post test values are not significant.
}

\title{
Referral and Accessibility of Health Facilities
}

Table 4

Referral and Accessibility of Health Facility: Postabortion Patients

\begin{tabular}{||c|c|c|}
\hline \multicolumn{1}{|c|}{ Variable } & Pre-test & Post-test \\
\hline \hline Referred by ${ }^{*}$ & $(\mathrm{n}=519)$ & $(\mathrm{n}=517)$ \\
Self & 61.5 & 51.5 \\
Private & 29.9 & 34.4 \\
Public & 6.9 & 11.6 \\
Daya & 1.7 & 2.5 \\
\hline Accompanied by & $(\mathrm{n}=508)$ & $(\mathrm{n}=497)$ \\
Husband & 61.0 & 60.0 \\
Others & 33.9 & 35.8 \\
Unaccompanied & 5.1 & 4.2 \\
\hline Distance between home & $(\mathrm{n}=519)$ & $(\mathrm{n}=517)$ \\
and hospital & \\
$\leq 5 \mathrm{Km}$ & 36.0 & 38.7 \\
$>5 \mathrm{Km}$ & 64.0 & 61.3 \\
\hline
\end{tabular}

Source: - Patients' Exit Interviews

a Medical Record

* Differences between pre and post test are significant $\mathrm{P}<0.001$

Table (4) presents data on referral and accessibility of health facilities for postabortion patients. About $62 \%$ of patients in the pre-test sought medical treatment themselves and were self referred to the health facilities. This proportion is lower in the post-test (about 52\%). The percentage of those referred to the hospital in the pre test survey by a private facility is about $30 \%$ compared to referrals by public facility (about $7 \%$ ). On the other hand, percentages in the post-test were about $34 \%$ and $12 \%$, respectively. These differences in pre and post test findings are statistically significant. Dayas referred only about $2 \%$ of postabortion patients to the hospitals in pre and post surveys.

It is interesting to note that about two-thirds of the post-abortion patients in pre and post test surveys were accompanied by their husbands. Others were accompanied by a mother, a sister or in-laws. 
Overall Table (4) indicates reasonable accessibility to health facilities. More than one third of patients in both pre and post test surveys traveled $5 \mathrm{~km}$ or less to reach a health facility. It

About $61 \%$ in the pre-test and $51 \%$ in the post-test were self referred. $61 \%$ were accompanied by their husbands and almost the same percentage traveled for more than $5 \mathrm{~km}$ to reach a health facility.

is noteworthy that accessibility to study sites in upper Egypt was found to be higher than sites in lower Egypt in both surveys. This may be due to the preponderance of cases in Shatby hospital (in Lower Egypt) which has a very large catchment area. Additionally few hospitals from lower Egypt were included in this study.

\section{QUALITY OF CARE FINDINGS}

The quality of care conceptual framework developed by Bruce (1990) was used in this study. It collected data on the following elements of service quality:

- Technical competence of health providers

- Interpersonal relations between providers and patients

- Provider-Patient Information exchange

- Constellation of services (linkage with family planning services)

For each element, three criteria were examined:

- Readiness to provide quality service;

- Quality of service provided;

- Quality of services received by the patient.

This framework will be used throughout the following sections which describes the major findings. It may be noted that several indicators from the medical record are used to describe process aspects of the quality of service provided.

\section{A. Physician's Technical Competence: Postabortion Care and Family Planning 1. Knowledge of complications, Sequelae and Warning Signs}

The Staff Interview Form included a question on whether the physician received specialized training in postabortion care. Findings of the pre-test interviews with physicians showed that the vast majority $(90.6 \%)$ received no specialized training in postabortion care by vacuum aspiration. The effect of the study intervention is reflected in the post-test, as approximately $46 \%$ of the medical staff reported training in vacuum 
aspiration. This is in addition to continuous transfers and rotations of the staff at the different study sites. This figure may be an underestimate as it is highly probable that some physicians who were exposed to on the-job-training may not perceive it as specialized training because it was not formal. In addition, only those staff who routinely provide postabortion treatment were trained whereas most of staff were interviewed and aware of the program to improve services. 
Physicians were asked about the early complications a woman can experience after having an abortion or miscarriage. Figure 1 shows the positive effect of training on the improved knowledge of those who said that they attended the specialized training course during the study intervention period. In both the pre- and post-test surveys most of the physicians ( $85 \%$ and $95 \%$ ) were able to cite hemorrhage as a possible complication. The increase in knowledge

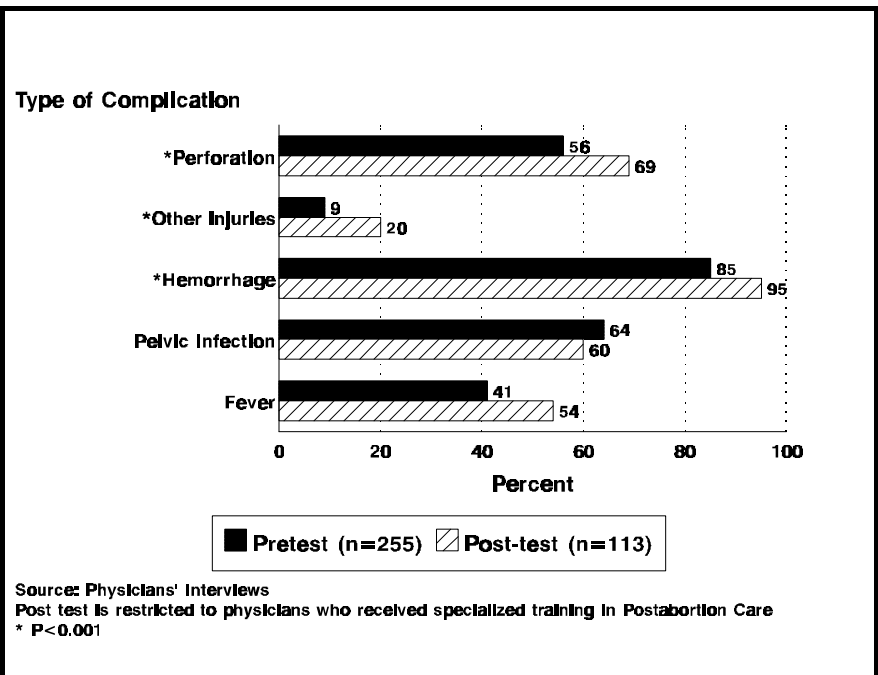

Figure 1: Physicians knowledge of early complications of abortion.

in the post-test was statistically significant with regard to citing hemorrhage, perforation of uterus, other injuries and fever. Whereas the difference was insignificant regarding knowledge of pelvic infection.

Figure 2 shows that physicians' knowledge of the long term sequelae of abortion were deficient before the study. Although some improvements were achieved as a result of the training in the post-test, their knowledge about the adverse sequelae of abortion still needs to be strengthened.

Prior to the interview, $42 \%$ of the medical staff reported that a postabortion patient has an increased risk for incompetent cervix and 19\% reported ectopic pregnancy. In addition, 15\% reported an increased risk of $\mathrm{RH}$ isoimmunization after an abortion. After the

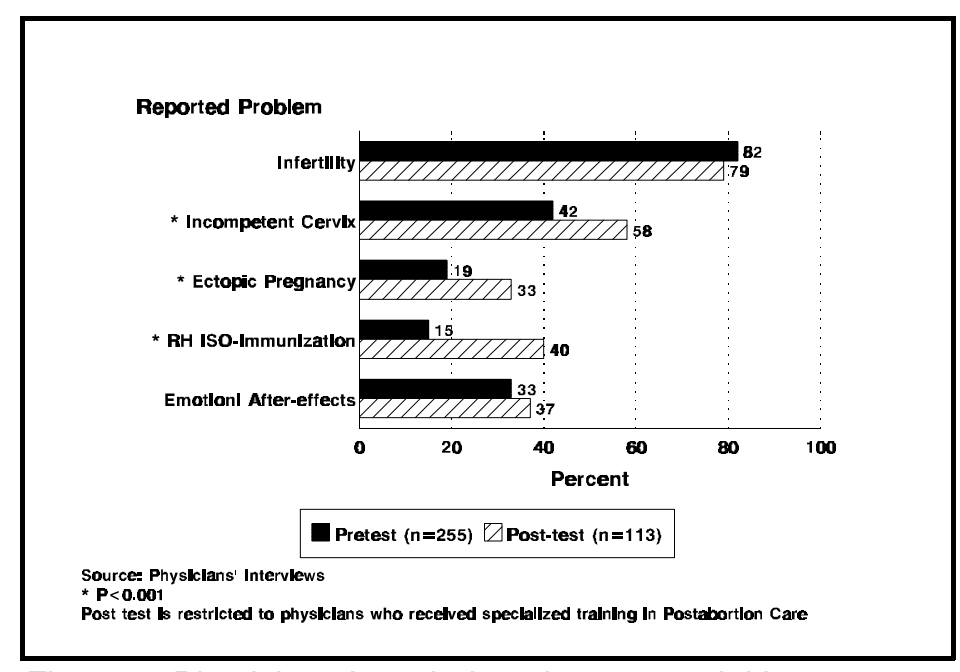

Figure 2: Physicians knowledge about potential long-term (late sequelae) of abortion. training program, 58\% reported 
incompetent cervix, 33\% cited ectopic pregnancy and about $40 \%$ mentioned $\mathrm{RH}$ isoimmunization. These differences in pre and post test were statistically significant.

Physicians were also asked about warning signs of complications after having an abortion or a miscarriage that a woman should be aware of. Figure 3 shows that after the intervention, physician's knowledge about warning signs of complications improved slightly. Exceptions are both the improved knowledge about unusual bleeding

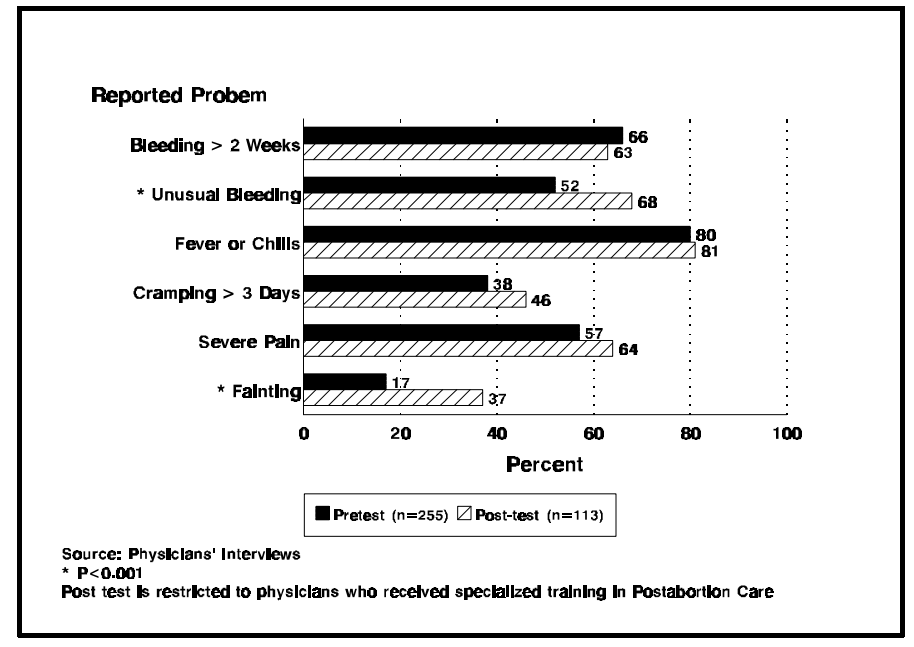

Figure 3: Physicians knowledge about warning signs of complications of abortion. which increased by 16 percentage points and knowledge about fainting which increased by 20 percentage points in the post test and these differences were statistically significant.

\section{Surgical Techniques and Pain Control Medication Used}

\section{a) Surgical Techniques}

The medical record included information on the type of procedure and the type of anesthesia provided to postabortion patients. Figure 4 shows a dramatic change in the type of surgical procedure used in the treatment of incomplete abortion. Prior to the study intervention, about $98 \%$ of postabortion patients were treated with dilatation and curettage (D\&C), while the rest were treated with MVA (mostly in Menia University

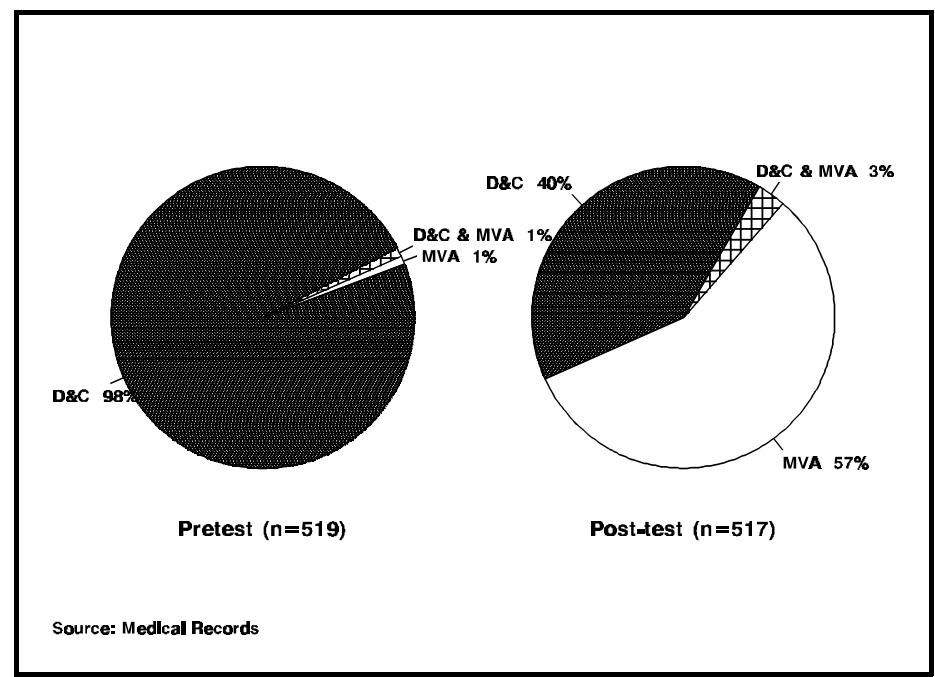

Figure 4: Type of surgical procedure used for the treatment of postabortion patients. 
hospital where its medical staff were trained on the use of MVAs during the pilot study conducted in 1994). After the study has introduced the MVA technique, the percentage of postabortion cases treated with D\&C dropped to about $40 \%$ whereas the percent of cases treated by MVA increased to $57 \%$. About 3 percent of patients were treated with a combination of $D \& C$ and vacuum aspiration, denoting a phase of experimentation with the new surgical technique by the recently trained physicians. Thus, MVA use increased from $2 \%$ to $57 \%$ of cases as a result of the study's intervention.

\section{b) Pain Control Medication}

The protocol used in the study intervention for improving the medical treatment of postabortion patients recommended the use of Manual Vacuum Aspiration under local anesthetic (paracervical block) with pre-operative sedatives and/or analgesics used when indicated.

Figure 5 shows that during the pre-test, general anesthesia was routinely used with the majority of cases $(85 \%)$, whereas $14 \%$ of the patients did not receive any form of anesthesia. It should be noted that conducting the surgical procedure with no pain control medication was largely found in hospitals other than University hospitals (26\%). The comparable figure for University

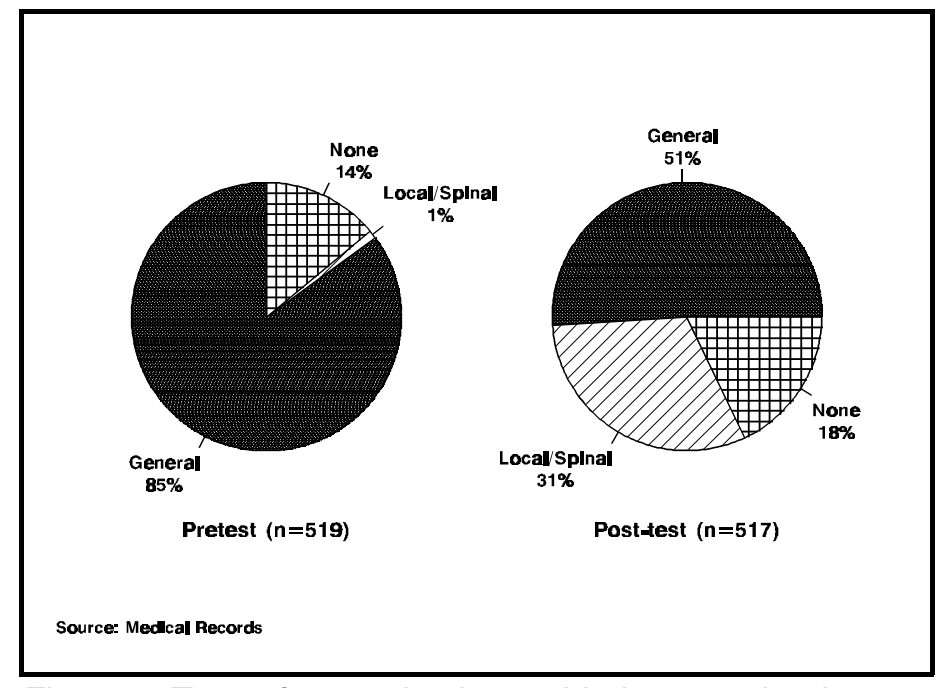

Figure 5: Type of anaesthesia provided to postabortion patients. hospitals was only $4.2 \%$. After the intervention, the use of general anesthesia dropped to $51 \%$ whereas the use of local anesthesia increased from $1 \%$ to $31 \%$. The continued use of general anesthesia in the post test may be attributed to several factors. Physicians recently trained may not feel comfortable dealing with patients who are conscious. In addition, some postabortion cases with certain medical conditions may require treatment under general anesthesia. Some physicians also reported that some 
patients e.g. primi parous women are too nervous during the procedure and therefore need general anesthesia. Some others reported patients' preference for total unconsciousness during the procedure especially in some lower Egypt hospitals. 
Through patients' exit

interviews, post-abortion patients were asked about the pain they experienced during and after the surgical procedure. Figure 6 shows that patients who reported extreme pain during the procedure increased from about $11 \%$ in the pre-test to about $15 \%$ in the posttest (not a significant difference). Those experiencing moderate pain during the procedure increased from about $5 \%$ in the pre-test to about $27 \%$ in the posttest while those reporting no pain decreased from about $84 \%$ in the pre-test to about $58 \%$ in the post-test. This may suggest relation to the type of pain control medication used. It is to be noted that the study indicated statistical significance $(p<0.001)$ in pain experienced by patients in pre and post test surveys for the last two groups.

\section{Physicians Knowledge about Resumption of Fertility}

Physicians were asked about the timing of return to fertility after having spontaneous or induced abortion. It is known that a woman's fertility is resumed almost immediately after an abortion (usually within 2 weeks after a first-trimester abortion), (IPAS, 1996). Figure 7 indicates fairly weak knowledge about this important technical information among all physicians in

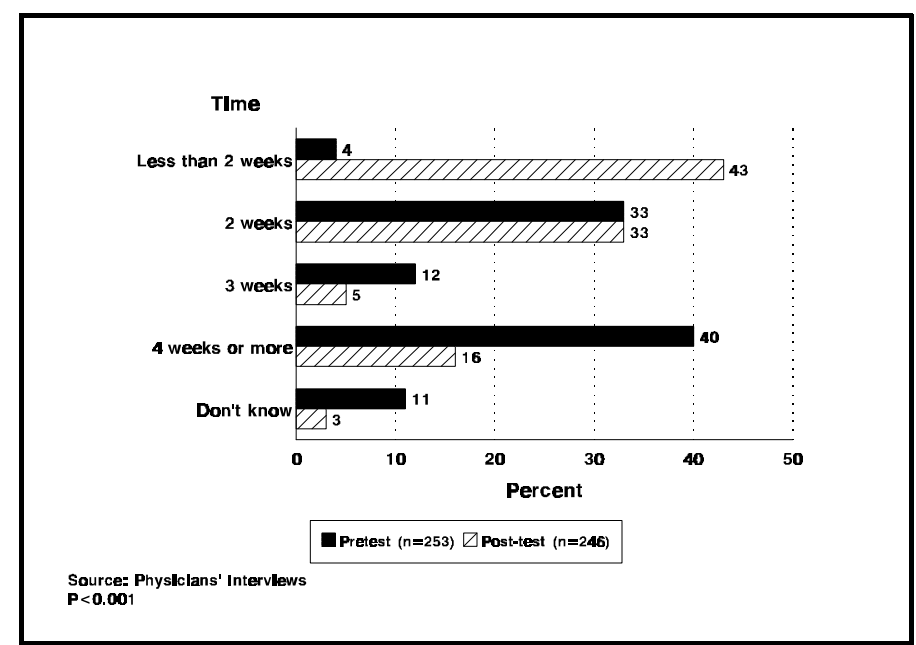

Figure 7: Physicians knowledge about timing (in weeks) when postabortion patients resume fertility. the pretest survey as only $4 \%$ were able to report the correct answer. 
After the intervention this percentage increased to $43 \%$. Resumption of fertility after two weeks was reported by about one third of physicians in both pre and post test surveys. It is important to note that the number of physicians in this analysis represents the whole studied group in the post test.

\section{Physicians Attitudes and Practices with regard to Improved Clinical Practices}

This section highlights feedback of physicians in the post test survey about the training course on improved postabortion care, their opinions, attitudes and use of Manual Vacuum Aspiration (MVA) compared to dilatation and curettage (D\&C) in the management of incomplete abortion.

Physicians who attended the training course were asked if they received the UNFPA supplied training materials that consisted of IPAS reference manuals and scientific papers. All except very few $(98 \%)$ reported receiving these materials. $38 \%$ of those who received the materials had actually read them, $59 \%$ read part of the materials and $3 \%$ did not read any at all (not shown in a figure).

Physicians were also asked about the usefulness of the training materials. Nearly all of trained physicians reported that these materials were either very useful $(45 \%)$ or somewhat useful (53\%), with few stating that it was not useful (2\%) (not shown in a figure). The post test survey also assessed physicians feedback regarding both theoretical and practical aspects of the training. Findings indicated that the receptivity for practical training among physicians was very high, with $76 \%$ of

Nearly all of trained physicians reported that the training materials were either very useful (45\%) or somewhat useful (53\%). Meanwhile, $76 \%$ of trained physicians reporting that practical training was very useful.

trained physicians reporting that practical training was very useful. Conversely, only about $53 \%$ found the theoretical training very useful. Other remaining answers to both aspects of training were "somewhat useful". Physicians were furthermore asked about ways to improve future training courses. More than one third of respondents suggested placing more emphasis on the practical sessions while one third were satisfied with the training with no modifications (not shown). 
Physicians who said that they were aware of MVA $(n=235)$ were asked about their views regarding the use of MVA versus $D \& C$ in the management of early gestational age of incomplete abortion in terms of risk of complications, its ease of use and its effectiveness (Figure 8). About three quarters of physicians interviewed (among those who were aware of MVA) reported that complications were lower with MVA than if patients were managed with D\&C. $61 \%$ stated that MVA was

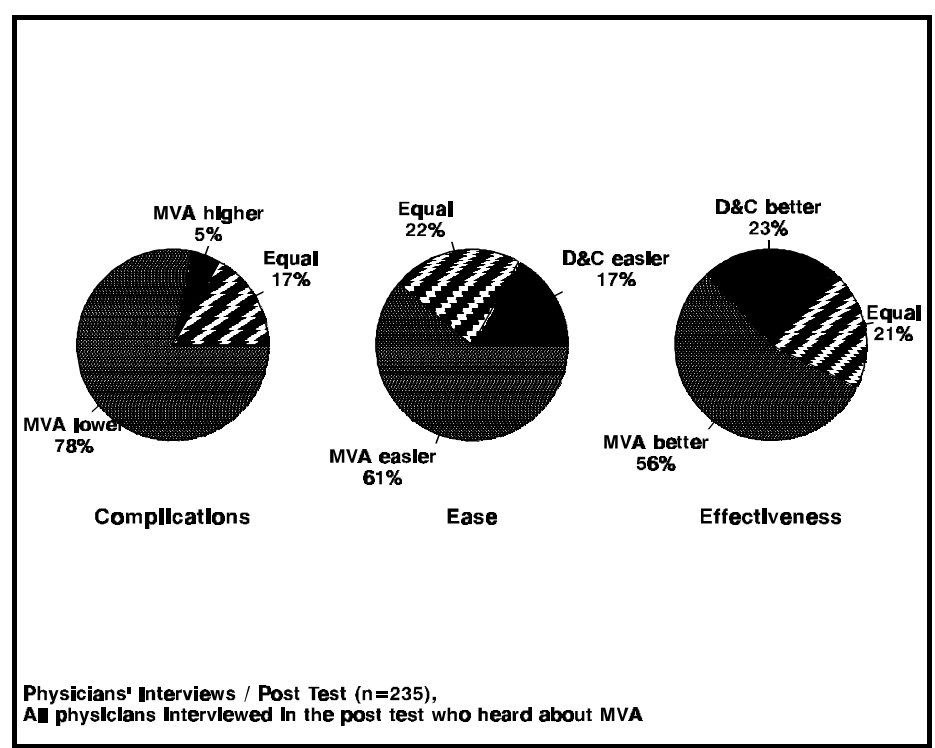

Figure 8: Physicians views regarding use of MVA vs. D\&C for treatment of incomplete abortion at early gestational age.

easier to use and $56 \%$ mentioned that it was more effective than D\&C. However, in terms of ease of use and effectiveness still $17 \%$ and $23 \%$ respectively think that MVA was inferior to $\mathrm{D} \& \mathrm{C}$.

In general, there is evidence that physicians working in the study hospitals were interested in the new technology (the use of MVA) as $95 \%$ reported in the post test that they have seen MVAs used by other physicians, $64 \%$ reported they have used it themselves and $63 \%$ trained other physicians on its use (Figure 9).

As mentioned earlier, the training course aimed at introducing the use of MVA as part of a holistic perspective of improving health care of postabortion patients. Thus it stressed the use of MVAs in cases of first trimester abortions under local anesthesia

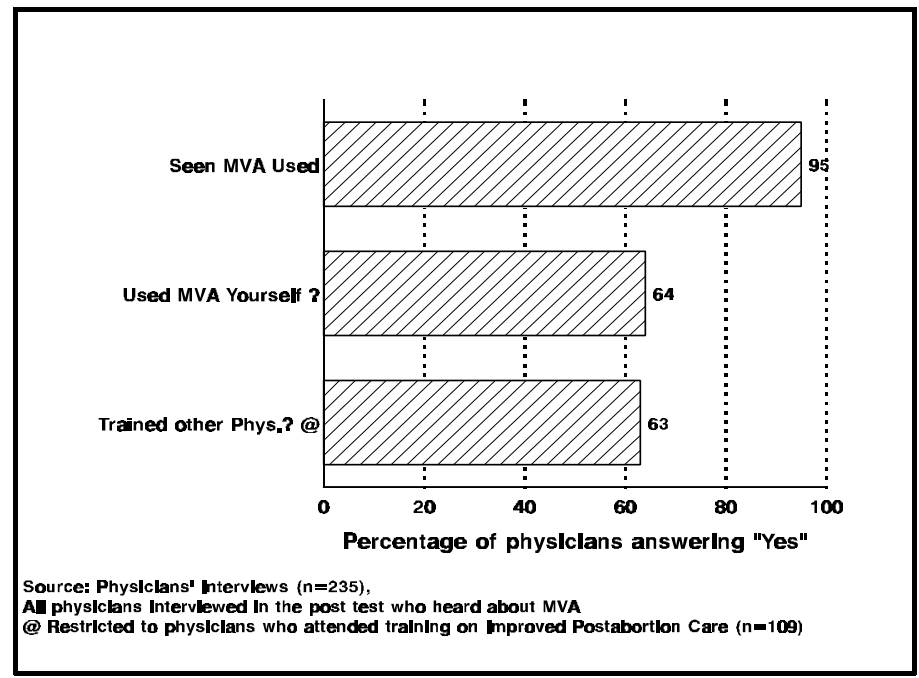

Figure 9: MVA clinical practice: physicians' reports (post test) rather than general anesthesia to minimize the risk of complications. 
In the post-test survey all physicians were asked about the frequency of use of local and general anesthesia in the past 4 months. Figure 10 indicates a movement towards more frequent use of local anesthesia, reflecting adherence to clinical protocols established by the study intervention (about $43 \%$ of physicians reported use of local anesthesia in about three quarters of cases or more while only $19 \%$ of physicians reported use of general anesthesia in three quarters of cases or more).

\section{B. Interpersonal Relations}

Postabortion patients seeking medical treatment are admitted, in most cases, to the hospital under severe emotional stress and physical discomfort. The positive interaction between health providers and patients help ease patients anxiety and concern. Thus, when asked "what can you do during the procedure to reduce patient's anxiety?", it was interesting to observe that $93 \%$ or more of the physicians cited reassurance in both pre and post-test surveys (Figure 11). Meanwhile study findings reflect other concrete improvements in specific steps to reduce patients' anxiety in the post test. For example, the percent reporting

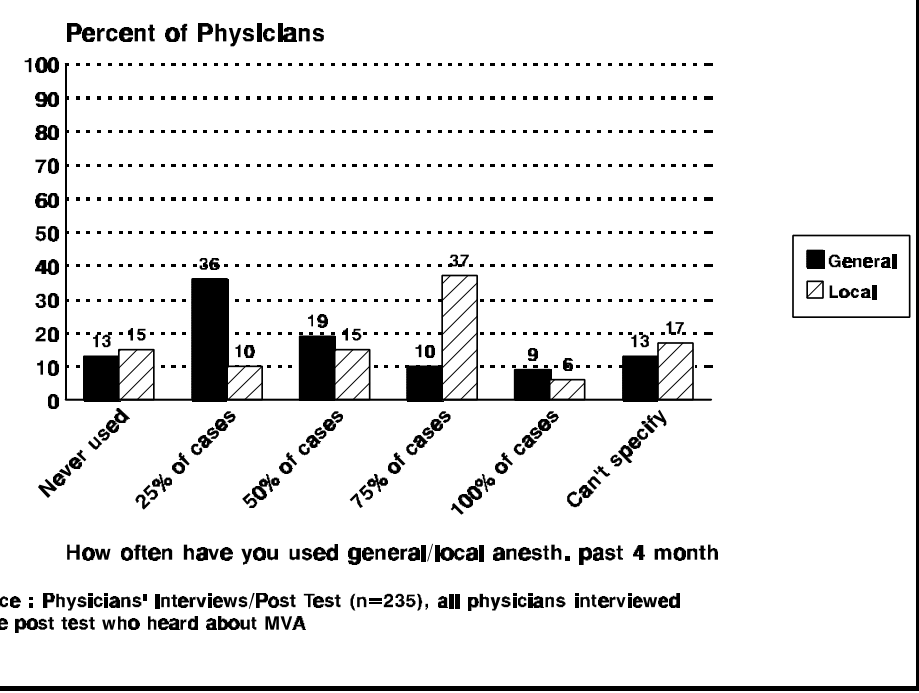

Figure 10: Physicians reports on their use of anesthesia in the past four months.

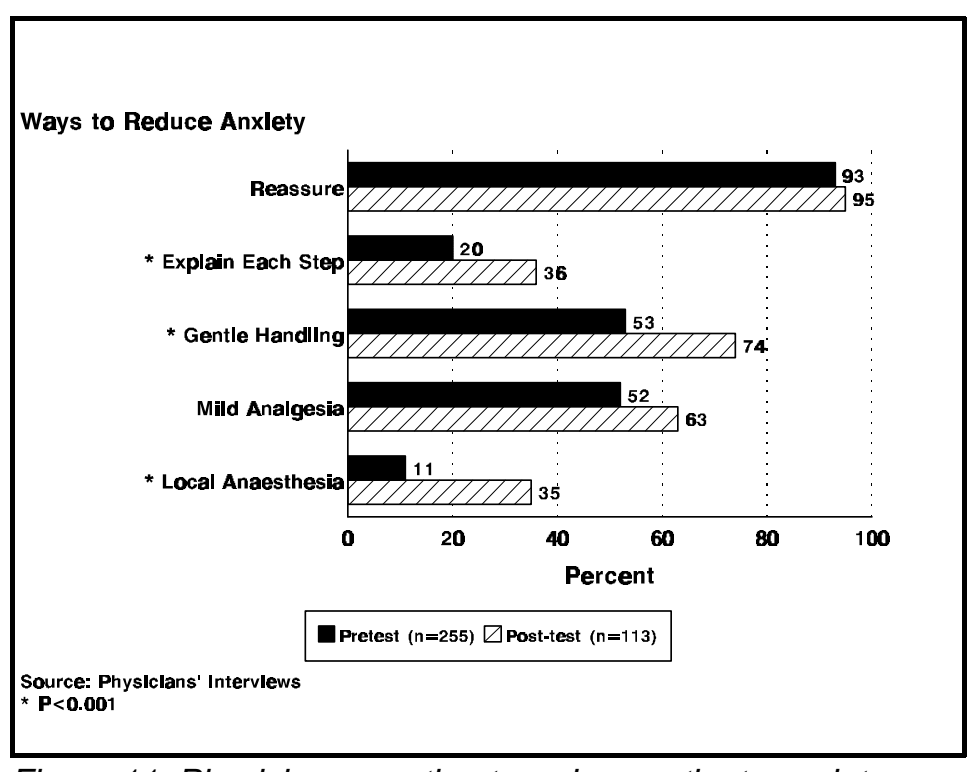

Figure 11: Physicians reaction to reduce patients anxiety during the abortion procedure. 
explaining each step in the consultation increased from $20 \%$ in the pre test to $36 \%$ in the post test. Those who reported gentle handling increased from $53 \%$ to $74 \%$. Furthermore, the percentage of physicians mentioning that mild pre-operative analgesia and local anesthesia could be of help in reducing patients' anxiety increased from $52 \%$ and $11 \%$ in the pre test to $53 \%$ and $35 \%$ respectively in the post test. These differences are statistically

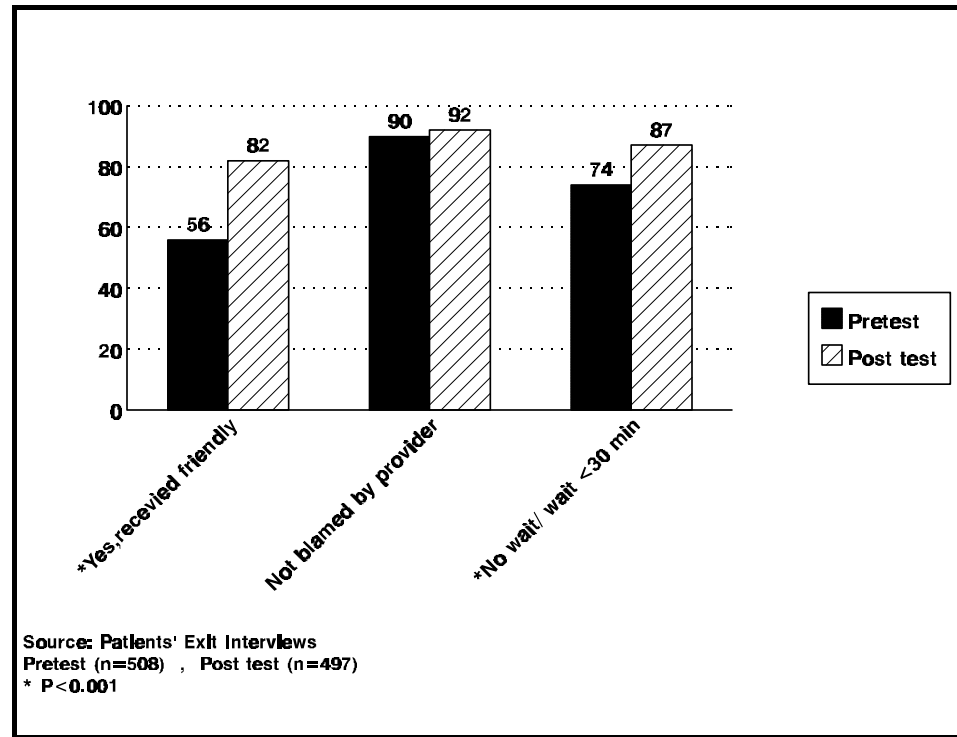

Figure 12: Selected indicators of interpersonal relations: reports of postabortion patients. significant.

Considering patient perspectives, Figure 12 presents selected indicators of interpersonal relations based on postabortion patients reports at the exit interview. The proportion of patients who reported that the provider who received them was friendly increased from $56 \%$ in the pre-test to $82 \%$ in the post test. Postabortion patients were also asked if any of the service providers blamed them because of their medical condition. The majority in both pre and post test surveys reported "No". In addition, postabortion patients were asked about waiting time between admission and receiving medical consultation. About $74 \%$ reported in the pre-test that they either received medical consultation immediately (no waiting time) or waited for less than half an hour. This proportion increased to

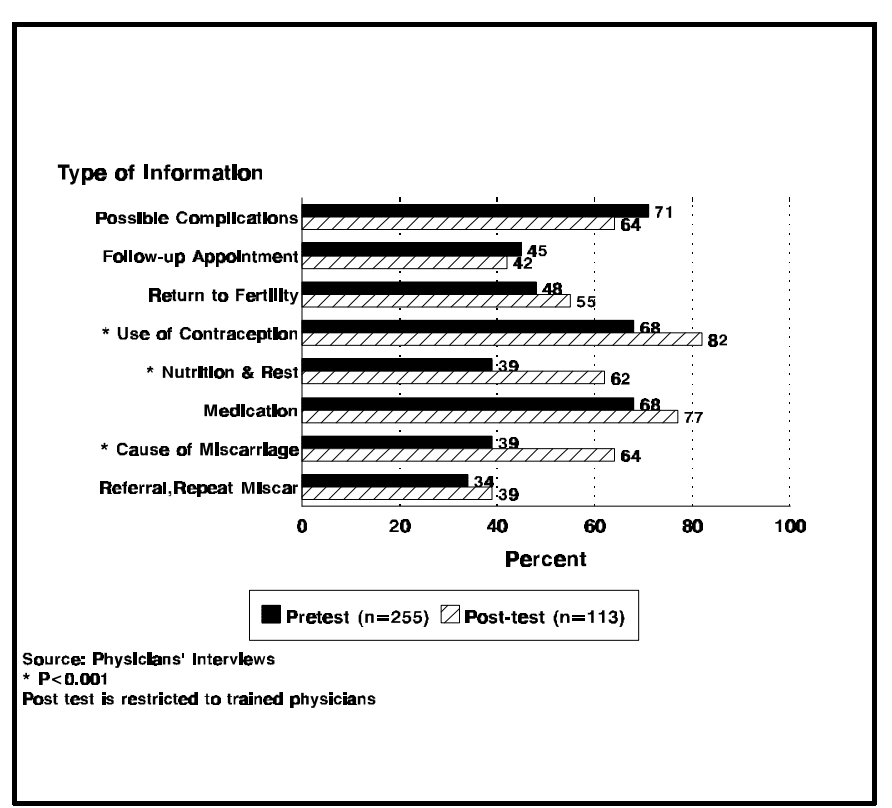

Figure 13: Type of information physicians think that postabortion patients should know before discharge. 
$87 \%$ in the post-test. Differences in the first and last indicators were statistically significant.

\section{Information Exchange}

Figure 13 presents pre and post test findings on physicians knowledge about the type of information postabortion patients should know before discharge. Overall, the data reflected modest knowledge about this particular aspect of information. However, major improvements in physician's knowledge were observed with regard to the need to explain cause of miscarriage to postabortion patients (increased from 39\% in pre-test to $64 \%$ in post test), nutritional requirements and rest needed for patients (39\% in pre-test / $62 \%$ in post test) and the need to use contraception ( $68 \%$ in the pretest $/ 82 \%$ in post test). These differences were statistically significant. Some other smaller improvements in physicians' knowledge about important follow-up information were also observed with regard to use of medications ( $9 \%$ increase in the post test), time patients resume fertility (7\% increase) and referral for repeat miscarriage ( $5 \%$ increase). However, no improvements were observed for other aspects.

The study intervention activities stressed the importance of the clinical management of postabortion patients and highlighted the importance of postabortion counseling and information exchange.

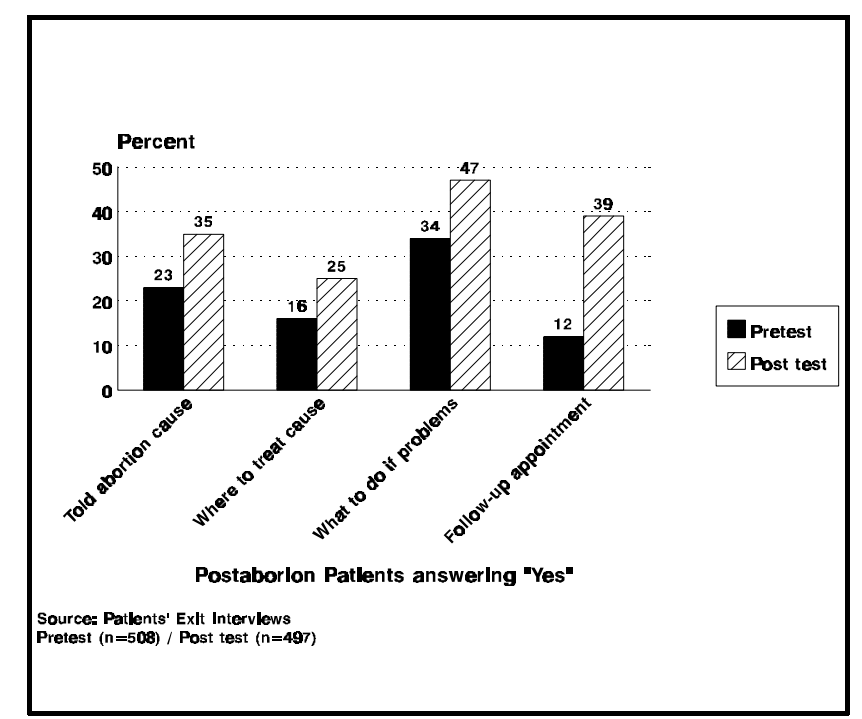

Figure 14: Follow-up information provided to postabortion patients.

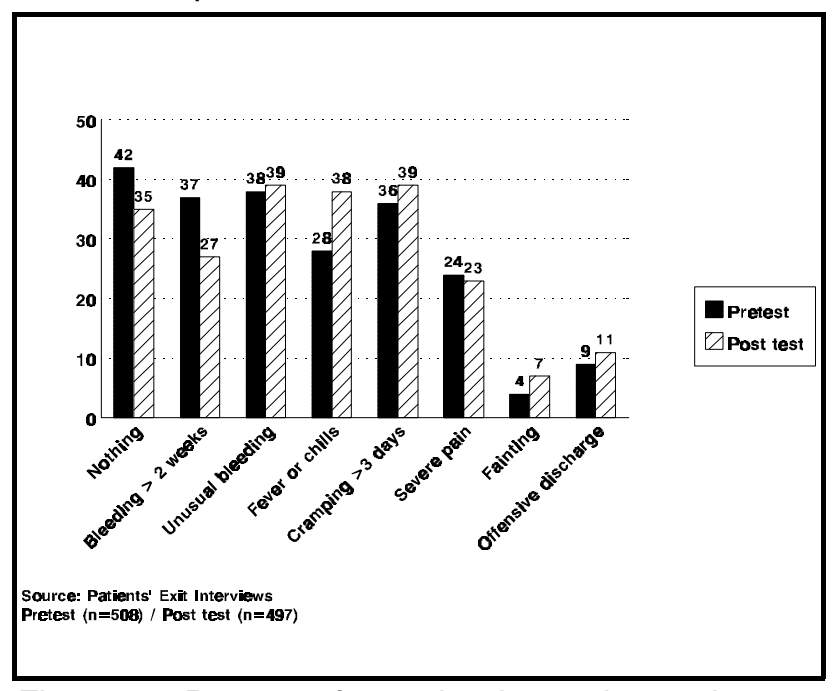

Figure 15: Percent of postabortion patients who received information about possible complications. 
Figure 14 shows improvements in follow-up information provided to postabortion patients in the post test compared to pre-test. More postabortion patients were told about the cause of abortion (12\% increase) and where to go to investigate or treat these causes ( $9 \%$ increase). Furthermore, more postabortion patients reported being told about what to do if problems occur after discharge (13\% increase) and that they were told about a follow-up visit (27\% increase).

Figure 15 also shows additional information received by postabortion patients on possible complications that may occur after abortion that the patient should be aware of. Overall, patients received more information in the post test compared to the pre test on possible bleeding, fever or chills, cramping more than 3 days, fainting and offensive discharge. However, in most cases the increase in knowledge exchange has been either small or modest and there is still clearly room for more improvement.

The study's intervention had also some impact on the role the paramedical staff can assume in counseling and

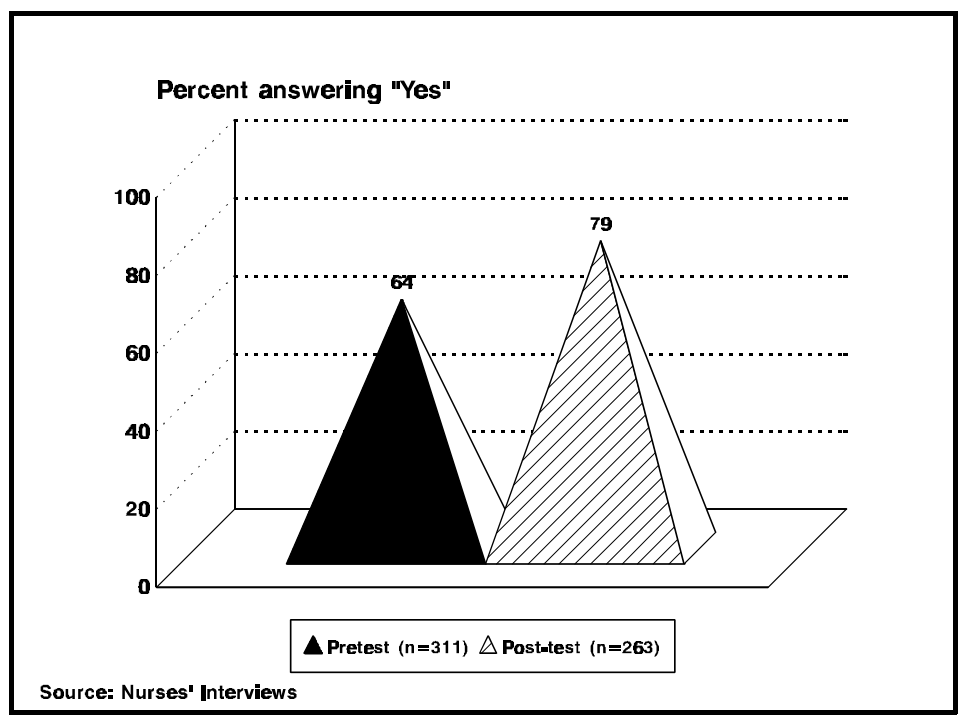

Figure 16: Postabortion family planning counseling by nurses. comforting postabortion patients. In this regard, nurses were asked whether they get any chance to talk to postabortion patients about family planning. The percentage of nurses reporting "Yes" increased from about $64 \%$ in the pre-test to about $78 \%$ in the post test. That difference was statistically significant (Figure 16).

When patients were asked in their exit interviews if they received information at the hospital on family planning, about $35 \%$ in the pre-test and $47 \%$ in the post test gave affirmative answer, (the difference is statistically significant). Those who haven't received information on family planning were further asked if they were told where to go to get information. Only about $5 \%$ of these patients answered "yes" in pre-test compared to $24 \%$ in the post test (not shown in a figure). 


\section{Utilization of Family Planning Services}

Figure 17 shows several indicators on family planning history among postabortion patients from both the Medical Records and the Patients' Exit Interviews. About 51\% of postabortion patients in the pre test reported ever use of contraceptive methods (vs. $47 \%$ in the post test). These figures are less than the $70 \%$ ever use of methods among married women reported in the 1995 EDHS. $19 \%$ of postabortion patients in the pre-test and $17 \%$ of patients in the post test reported that they

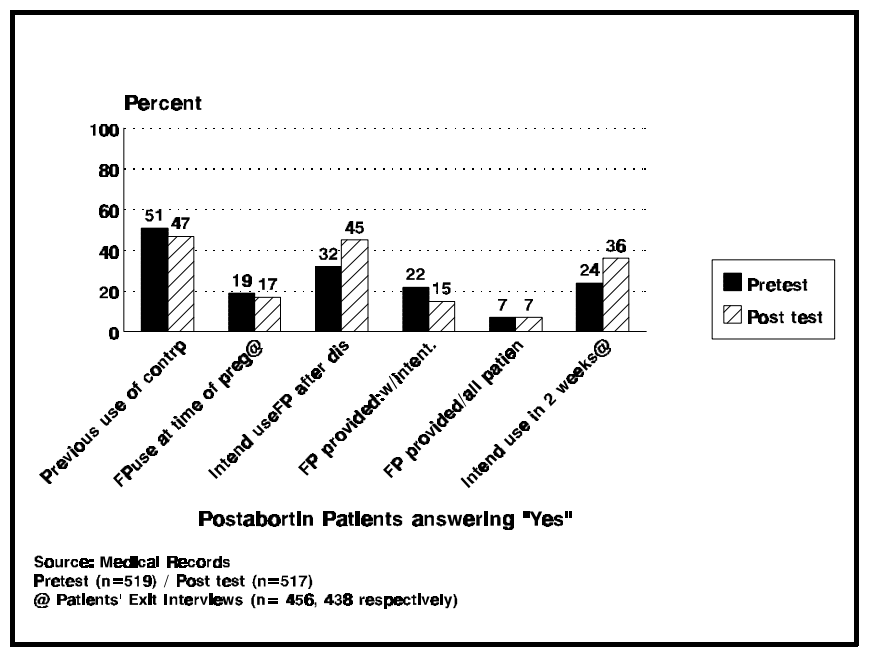

Figure 17: Family planning history among postabortion patients. became pregnant (the index pregnancy) while using a contraceptive method. The survey findings indicated that the most common two methods used by those women were the pill and IUD (not shown). About one third of postabortion patients in the pre-test and close to one half in the post-test reported intention to begin using a contraceptive method during the postabortion period. Among them, $22 \%$ in the pre-test and $15 \%$ in the post test were actually provided a method before discharge. However, only $7 \%$ in both pre and post tests of all postabortion patients who received treatment from abortions or miscarriages were provided a method before discharge. The last indicator presented in Figure 17 shows the percent of postabortion patients who intend to begin using a family planning method within two weeks from discharge. Data on this indicator show a 12 percentage points increase between the pre and the post test $(24 \%$ vs. $36 \%$ respectively). 


\section{Satisfaction of Postabortion Patients with Service Received}

Through the patient exit interview postabortion patients were asked about their perception of the service they received at the hospital. Figure 18 shows substantial improvement in patients' satisfaction with quality of service received. Close to threefourths of patients in the post test described the service they received as "excellent" compared to less than one half of patients

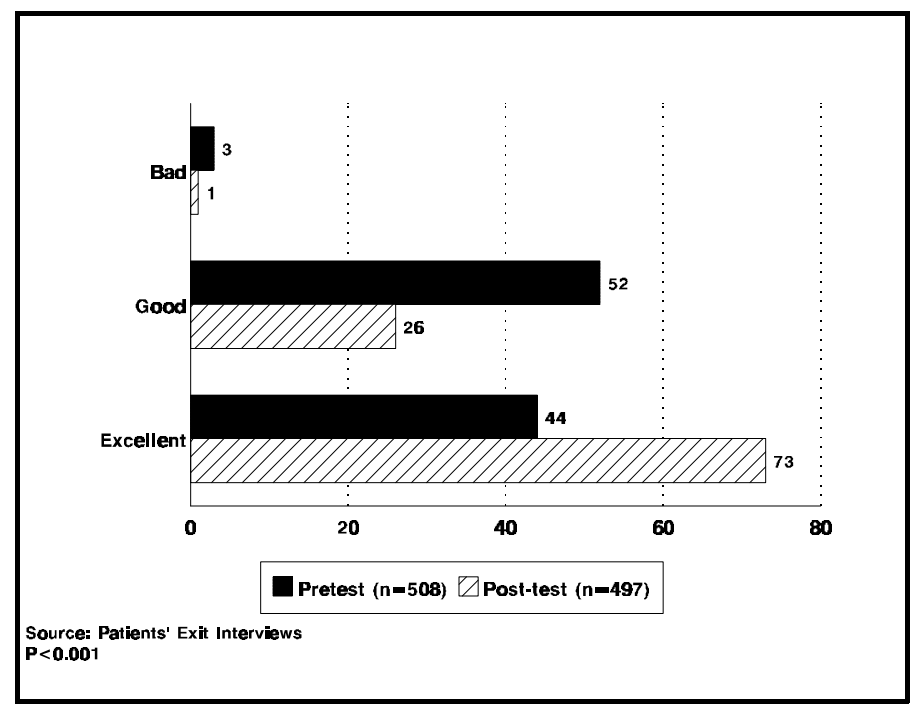

Figure 18: Postabortion patients satisfaction with the quality of service received. in the pre-test (45\%). Few patients, however, reported that the service they received was "bad" in both pre and post test surveys.

\section{Duration of Hospital Stay}

The medical records collected data on duration of hospital stay for postabortion patients. The study findings indicate that the mean duration of hospital stay for all patients who were admitted in the ten study sites for a continuous one month period in the pre test was 21.3 hours, and in the post test was 20.6 hours. The difference between these two means is not statistically significant. It may be noted here that the duration of hospital stay could be affected by hospitals policies and protocols concerning treatment, management and discharge of postabortion cases. Some of the hospitals do not allow patients' discharge until next day morning round when physician in-charge signs the necessary papers allowing the patient to leave the hospital. 


\section{Overview}

This report presents findings of a study that launched an expansion of improved postabortion care introduced through a pilot study in two hospitals in 1994. The program for scaling up improved postabortion care services included ten university and public sector hospitals in both Lower and Upper Egypt.

The study intervention focused on the use of Manual Vacuum Aspiration instruments (MVA) combined with local anesthesia. Physicians were also instructed in infection control practices and counseling postabortion patients about their medical condition, family planning use and related reproductive health care services (e.g. referral for repeat miscarriage). This training program was followed by on the job training for all service providers in the ten sites for a continuous four month period to implement the new clinical practices and standards of care launched during the study's intervention.

Findings presented in this report are based on data collected through interviews with medical providers who are involved in providing care to postabortion patients (physicians, nurses and social workers) as well as exit interviews with all consenting postabortion patients about the quality of care they received. In addition, the study utilized abstracts of Medical Records to described selected medical, sociodemographic and reproductive history profile of these patients.

Within a quality of care conceptual framework, this report examined physicians technical knowledge and their clinical practices related to postabortion care as well as their communication skills with patients. The study also examined aspects of care received by postabortion patients based on women's self reports before discharge.

The study findings indicate that prior to the study professional opportunities to develop new skills or to improve clinical practices for complete management of postabortion patients were not common. Very few physicians received specialized training on postabortion care (9.4\%), being mostly University hospitals physicians. After the study, $46 \%$ of physicians reported receiving that type of training.

Using the WHO abortion classification guidelines for classifying morbidity 
related to abortion the findings showed that in both pre and post test phases, slightly more than one half of abortion cases (55\%) could be classified as spontaneous abortions, while the remaining cases were classified as likely induced (certainly, probably and possibly induced). These findings together with other findings reflect that a considerable proportion of postabortion patients still desire more children (more than one half) highlight the need to continue and strengthen adequate counseling for women experiencing spontaneous abortion as part of improved postabortion program to help them achieve their reproductive goals.

The study examined physicians technical knowledge and their clinical practices related to postabortion care. Findings reflected moderate improvements in physicians technical knowledge about early complications and long term sequelae of abortion as well as warning signs of complications after having an abortion or miscarriage. Thus, there is still room for more improvements in their technical knowledge through continuous in-service training.

The study findings indicate substantial change in the type of surgical procedure used in treatment of incomplete abortion. Prior to the study intervention, about $98 \%$ of postabortion patients were treated by dilatation and curettage (D\&C). After the study's intervention has introduced the MVA technique, the percentage of postabortion patients treated with D\&C dropped to about $40 \%$ whereas cases treated by MVA increased to $57 \%$.

Additionally, prior to the study, general anesthesia was routinely used in the majority of cases (85\%) with very little use of other types of pain control medications. The protocol used in the intervention recommended use of MVA under local anesthesia, with pre-operative sedation and/or analgesics given when indicated. Thus, use of general anesthesia dropped to $51 \%$ of cases whereas the use of local anesthesia increased from $1 \%$ to $31 \%$ of cases.

Findings also indicate that prior to the study, information given to postabortion patients regarding aspects of their health care, possible complications of abortion and important follow-up information was somewhat deficient. Moreover, indicators on 
physician-patient interpersonal relations were rather weak, as reported by patients. As a result of the intervention activities these important aspects of care were moderately improved. However, it was obvious that there is still room for more continued training of physicians in such areas of social and behavioral aspects of patient management.

Though substantial success has been achieved in adopting the new protocol for pain control medication used, some physicians were still resistant to the idea of using local anesthesia. Some physicians reported shortage of some pain control medications as one of the reasons for using general anesthesia. Some others indicated that patients often request to "be asleep" during the procedure.

Indicators on counseling component of improved postabortion care as reflected by this study, indicate some evidence of change towards more personalized care though still need to be strengthened and stressed. In general, more concerted efforts are needed to introduce institutional changes that are conducive to more personalized care.

The role of nurses in management of postabortion cases is underutilized as indicated by pre test data. The present study resulted in an increased interaction between nurses and postabortion patients especially with regard to family planning counseling.

In general, the success and experience gained in improving postabortion care through this expansion is indicative of the possibility for continued and expanded training of MVA technology. Egypt is a unique case of preponderance of available service providers who constitute good national capacity to undertake training needed both at central and local levels. Training in improved postabortion care will result in D\&C use yielding to the use of the new MVA technique, improved quality of services, and increased family planning method use.

\section{Recommendations}

- Integrated efforts need to be exerted at both policy and program levels to institutionalize continued training on improved postabortion care, including the use of MVA under local anesthesia. Current training centers and institutions 
should assume responsibility in this important area.

- In order to successfully institutionalize routine use of MVA at service outlets, sustainable supplies should be made available at an affordable price. Service providers should also be encouraged to use it in their private practice by all means (exposure to training, procurement of supplies).

- A protocol for the use of MVA as a part of the accepted medical standards needs to be incorporated in the undergraduate and graduate medical curricula. This will ensure a minimum level of training for future generations of service providers.

- The role of MOHP is essential in securing necessary drugs and chemicals needed for pain and infection control procedures for use of the MVA instruments. A system should be developed for procurement of such medical requirements in consultation with the medical staff of the study sites.

- An effective mechanism needs to be developed for integrating family planning, postabortion care and other related reproductive health care services by reducing the logistics and administrative barriers.

- High emphasis should be placed on training of nurses involved in postabortion care. They have a pivotal role in contacting, communicating and comforting patients. It would be helpful if a simplified guide be designed in Arabic on description of MVA instruments, their advantages in treating incomplete abortion and their storage, sterilization and disposal requirement. Additionally, a simple guide to family planning counseling is needed. 


\section{BIBLIOGRAPHY}

Bruce, Judith "Fundamental Elements of Quality of Care: A Simple Framework". Studies in Family Planning, Vol. 21, No. 2: 61-91, 1990.

EFCS and Population Council ANE OR/TA Project. Improving the Counseling and Medical Care of Postabortion Patients in Egypt. (1995)

IPAS. MVA Trainer's Handbook, 1996 update.

WHO. Complications of Abortion Technical and Managerial Guidelines for Prevention and Treatment, Geneva: World Health Organization, 1995.

Yorde, Laura, Sarah Johnson, Judith Winker. "MVA Training Handbook" IPAS, Carrboro, North Carolina, 1993. 